\title{
Homogenization of piezoelectric planar Willis materials undergoing antiplane shear
}

\author{
Alan Muhafra, Majd Kosta, Daniel Torrent, René Pernas-Salomón and Gal Shmuel
}

Faculty of Mechanical Engineering, Technion -Israel Institute of Technology, Haifa 32000, Israel GROC-UJI, Institut de Noves Tecnologies de la Imatge, Universitat Jaume I, 12071 Castelló de la Plana, Spain

\begin{abstract}
Homogenization theories provide models that simplify the constitutive relations of heterogeneous media while retaining their macroscopic features. These theories have shown how the governing fields can be macroscopically coupled, even if they are microscopically independent. A prominent example is the Willis theory which predicted the strain-momentum coupling in elastodynamic metamaterials. Recently, a theory that is based on the Green's function method predicted analogous electro-momentum coupling in piezoelectric metamaterials. Here, we develop a simpler scheme for fibrous piezoelectric composites undergoing antiplane shear waves. We employ a source-driven approach that delivers a unique set of effective properties for arbitrary frequency-wavevector pairs. We numerically show how the resultant homogenized model recovers exactly the dispersion of free waves in the composite. We also compute the effective properties in the long-wavelength limit and off the dispersion curves, and show that the resultant model satisfy causality, reciprocity and energy conservation. By contrast, we show how equivalent models that neglect the electromomentum coupling violate these physical laws.
\end{abstract}

\section{Introduction}

Homogenization theories provide a simplified description of the physics of composites using a fictitious homogeneous medium that behaves the same in some appropriate average sense [5, 13, 20, 37, 46, 65, 67]. The simplified description shows that the behavior of certain composites can be essentially different from the behavior of their comprising constituents, in which case they are called metamaterials $[8,12,14,15,21,28,30,59,61,62,68,71]$. 
Central to the field of metamaterials in elastodynamics is the theory of Willis $[72,73,74$, 78], which was developed at the level of generality of random media, and relies on ensemble averaging to handle the randomness. Notably, the theory also applies when periodicity is present as a particular case. The theory shows that the effective response of a material point is nonlocal in space (i.e., depends on the state of other points) and time (i.e., depends on previous states). The theory further shows that the effective velocity may induce an effective stress, and that the effective strain may induce an effective momentum. These nonlocal couplings-which are absent in conventional materials - are now termed the Willis couplings. To date, the theory is still being refined and analyzed by Willis $[77,79,80]$ and others $[33,35,41,45,47,49,50,52,54,56,57$, $64,69]$. These theoretical studies are accompanied with experiments that measure and exploit the Willis coupling for applicational purposes [9, 26, 29, 32, 34-36, 38, 42, 53].

As mentioned, the first developments of the theory were for elastodynamics. Owing to the similarity in the wave equations of different physical media, the applicability of the Willis theory extends beyond elastodynamics, e.g., to acoustics, fluid dynamics and electromagnetism [43, 54, 75]. In the latter case, the theory predicts that the electric displacement is coupled with the magnetic field, and that the magnetic induction is coupled with the electric field [75]. Thereby, Willis theory agrees with what is already known in the photonics community as bianisotropic materials, described by the so-called magnetoelectric tensor [7, 23, 36, 57].

Most recently, Pernas-Salomón and Shmuel [49] have extended the approach of Willis to describe the dynamics of elastic composites that exhibit a coupling with thermal ${ }^{1}$-, electric- or magnetic fields at the microscopic scale. The generalized theory for piezoelectric constituents revealed additional couplings of Willis type between the electric displacement and the velocity, and between the momentum and the electric field. This result not only introduces new parameters to the design space of metamaterials, but also establishes a different route to manipulate elastic waves using nonmechanical stimuli. Indeed, the recent one-dimensional study by Pernas-Salomón et al. [51] has shown how the electromomentum coupling modifies the effective wave velocity and impedance, and generates asymmetry in the phase angle in addition to the asymmetry that the Willis coupling generates. Pernas-Salomón et al. [51] further showed how this asymmetry can be switched on and off by changing the electrical circuit boundary conditions.

The formulae of Willis [74, 75, 79] and Pernas-Salomón and Shmuel [49] are based on the Green's function of the relevant medium. However, the Green's function cannot be determined for most problems, and to obtain explicit expressions for the effective properties in the general case there is a need for other methods. In the elastic periodic case, it proved useful to use the plane wave expansion method [24, 58] as part of the averaging process [47, 48, 52]. Here, we extend this approach to obtain explicit expressions for the effective properties of periodic piezoelectric com-

\footnotetext{
${ }^{1}$ See also the work of Torrent et al. [70] on thermal materials.
} 
posites that undergo antiplane shear waves of Bloch-Floquet type. While this is the simplest setting that admits tensor-valued properties, spatial nonlocality and the number of properties that govern the medium render the problem very rich, as we show in the sequel. Its extension to problems that are more general is conceptually straightforward, however significantly more complicated from a technically perspective, and not pursued here.

The method in this work shares two key elements with the schemes of Willis [75] and PernasSalomón and Shmuel [49]. First, our definition for effective waves relies on the ensemble averaging of the microscopic fields, which reduces to volume averaging over the periodic part of the microscopic Bloch-Floquet waves. Second, we account for body forces and volume charge in our governing equations, and further introduce an eigenstrain in the microscopic constitutive relations. The main motivation for introducing these sources is mathematical: while the form they have in our formulation is challenging to access experimentally, if at all possible, this form delivers unique set of effective properties that is applicable for general frequency-wavevector pairs, not only for those that are related by the normal modes of the composite. (At the very basic level, without these sources the effective state variables become algebraically dependent; for an in-depth discussion on the issue of uniqueness, the reader is referred to Refs. [40, 45, 50, 79].) Importantly, the effective description that results from this approach satisfies basic physical laws, in contrast with other homogenization schemes, as we discuss in more detail later. This type of source-driven homogenization is rooted in the works of Fietz and Shvets [16, 17], Alù [4] and Willis [75].

The scheme is applied for two types of mixtures of PMMA matrix with PZT4 inclusions. The two mixtures differ by the shape of the inclusions: one mixture considers circular inclusions and the other considers inclusions without axial symmetry. The dispersion relations that the effective properties predict for free waves (i.e., the normal modes) are shown to be in perfect agreement with those of the original composites. We also apply our homogenization scheme in the long wavelength limit, where the medium can be approximated using local effective properties ${ }^{2}$, and outside the dispersion curves for arbitrary frequency-wavevector pairs. These calculations show that the effective properties that our homogenization scheme provides respect the mathematical conditions that follow from reciprocity, energy conservation and causality $[3,25,41,50,57,64$, 66]; the homogenized description should respect these conditions, since the components of the composite satisfy them. (Recent works actually seek to break these restrictions [44] using active components and digital control devices [9-11, 53, 81]. With such electronic circuits, researchers were able to engineer the injected energy and all the effective properties independently, thereby generating nonreciprocal, nonconservative response.)

As mentioned, in the absence of sources it is possible to define alternative homogenized mod-

\footnotetext{
${ }^{2}$ Milton et al. [36] were the first to introduce equations with local cross-coupling terms in elastodynamics, hence these equations can referred to as the Milton-Briane-Willis equations [39].
} 
els. By construction, these models may reproduce certain features in the macroscopic response of the composite, e.g., recover the dispersion curves, while neglecting the electromomentum coupling. We adopt the terminology in Refs. [4, 57] and refer to these models as equivalent models with equivalent properties. We calculate the equivalent properties on the dispersion curves and in the long wavelength limit, and show that they violate reciprocity and energy conservation. This observation supports our source-driven homogenization scheme, and specifically the need for the electromomentum tensor in the homogenized model to be physically admissible. It is in direct analogy with the observations of Alù [3] and Sieck et al. [57], that the bianisotropic and Willis tensors are needed in order to obtain physical effective properties of electromagnetic- and acoustic composites, respectively.

Our results are presented as follows. Sec. 2 summarizes the governing equations and introduces our definition of effective fields in periodic piezoelectric media. Sec. 3 develops the source-driven homogenization scheme for antiplane shear waves of Bloch-Floquet type in two-dimensional piezoelectric composites, using the plane wave expansion method. A numerical study is carried out in Sec. 4, followed by a summary of our main results and insights in Sec. 5 .

\section{Governing equations and effective fields}

The state of a piezoelectric medium is determined from the equations of motion in conjunction with the quasi-electrostatic approximation of Maxwell's equations. This approximation is valid when at the considered frequencies, the mechanical wavelengths are much shorter than the electromagnetic wavelengths. Accordingly, the equations are

$$
\nabla \cdot \boldsymbol{\sigma}+\mathbf{f}=\dot{\mathbf{p}}, \nabla \cdot \mathbf{D}=q, \nabla \times \mathbf{E}=\mathbf{0}
$$

where $\mathbf{f}$ is the body force density, $\boldsymbol{\sigma}$ is the Cauchy stress, $\mathbf{p}$ is the linear momentum, $\mathbf{D}$ is the electric displacement, $q$ is the charge density and $\mathbf{E}$ is the electric field. It is convenient to identically satisfy Eq. (1) $)_{3}$ using an electric potential $\phi$, such that $\mathbf{E}=-\nabla \phi$. The state variables of the medium are related through the constitutive relations

$$
\left(\begin{array}{c}
\boldsymbol{\sigma} \\
\mathbf{D} \\
\mathbf{p}
\end{array}\right)=\left(\begin{array}{ccc}
\mathbf{C} & \mathbf{B}^{\top} & 0 \\
\mathbf{B} & -\mathbf{A} & 0 \\
0 & 0 & \rho
\end{array}\right)\left(\begin{array}{c}
\nabla \mathbf{u}-\boldsymbol{\eta} \\
\nabla \phi \\
\dot{\mathbf{u}}
\end{array}\right)
$$

where $\mathbf{u}$ is the displacement field and $\boldsymbol{\eta}$ is an inelastic strain, the purpose of which is discussed in detail in Refs. $[49,50,75,79]$; the symbols $\rho, \mathbf{A}, \mathbf{B}, \mathbf{C}$ denote the mass density and the dielectric, piezoelectric and elasticity tensors, which are functions of the position $\mathbf{x}$ for a heterogeneous 
medium. In the sequel, we refer to the quantities in the right- and left column vectors as kinematicand kinetic variables, respectively. The objective of homogenization theories is to replace the description of the heterogeneous medium by a fictitious homogeneous medium that effectively behaves the same in some appropriate sense. This is carried out by first defining average effective fields, and then determining the effective constitutive relations between them. Here, we focus on time-harmonic Bloch-Floquet waves in periodic media, and adopt the definition of Willis [75], Alù [4] and the references therein for the average fields. Thus, we replace field variables of the form

$$
\zeta(\mathbf{x}, t)=\hat{\zeta}(\mathbf{x}) e^{i(\boldsymbol{\kappa} \cdot \mathbf{x}-\omega t)}
$$

by the effective fields

$$
\langle\zeta\rangle(\mathbf{x}, t)=\bar{\zeta} e^{i(\boldsymbol{\kappa} \cdot \mathbf{x}-\omega t)}, \bar{\zeta}=|\Omega|^{-1} \int_{\Omega} \hat{\zeta}(\mathbf{x}) \mathrm{d} \mathbf{x}
$$

where $\hat{\zeta}(\mathbf{x})$ is periodic with the same periodicity of medium, $\Omega$ is the periodic cell, and $\omega$ is the frequency. As discussed in Refs. [75, 79], this definition-which filters out the fast oscillations at the microscopic scale-delivers effective variables that satisfy exactly macroscopic balance equations that have the same form as Eq. (1). (See also Refs. [4, 45] for more arguments that support this definition.) Our goal is to develop a method for calculating a unique set of effective properties that relates the average fields in two-dimensional piezoelectric composites undergoing antiplane shear waves. To do so, we will use the plane wave expansion method in the averaging process, as described next.

\section{The homogenization scheme}

The basic idea in the following homogenization scheme using a plane wave expansion is simple: we represent the all field variables in their Bloch form and expand periodic functions in Fourier space; this allows us to extract from the governing equations the relations between the effective fields. This procedure is described next, for the problem of antiplane shear waves ${ }^{3}$.

We consider a piezoelectric composite that is periodic in the $\left(x_{1}, x_{2}\right)$ plane and uniform in the $x_{3}$ direction. The composite is driven by the presence of sources in the form

$$
s\left(x_{1}, x_{2}, t\right)=s^{0} \mathrm{e}^{i(\boldsymbol{\kappa} \cdot \mathbf{x}-\omega t)}, \quad s=\boldsymbol{\eta}, \mathbf{f}, q,
$$

\footnotetext{
${ }^{3}$ See Refs. $[18,19,55]$ for the use of the plane wave expansion to determine the normal modes of a fibrous electroelatic composites, and Ref. [6] for the purely elastic case.
} 
which generate antiplane shear waves in the composite; note that since $\left\{s^{0}\right\}$ are constants, it follows that $\langle s\rangle \equiv s$. As per the discussion in Sec. 1, we recall that these mathematical sources need not be experimentally realizable. Specifically, here form (5) demands the eigenstrain to be independent of the phase, and the charge to be controlled in time and space-difficult requirements to achieve in practice. However, as we show in the sequel, this formulation delivers derive a unique set of effective properties, satisfying reciprocity and energy conservation; this set, in turn can to describe the overall response of the composite in the presence of arbitrary physical sources.

Owing to the form of the prescribed sources, the displacement field has only one component in the direction of $x_{3}$, propagating in the $\left(x_{1}, x_{2}\right)$ plane; we denote this component by $u$. We represent the constitutive relations of the composite at each point in the following matrix form:

$$
\left(\begin{array}{c}
\sigma^{13} \\
\sigma^{23} \\
D^{1} \\
D^{2} \\
p
\end{array}\right)=\left(\begin{array}{ccc}
\mathrm{C} & \mathrm{B}^{\top} & 0_{2 \times 1} \\
\mathrm{~B} & -\mathrm{A} & 0_{2 \times 1} \\
0_{1 \times 2} & 0_{1 \times 2} & \rho
\end{array}\right)\left(\begin{array}{c}
u_{, 1}-\eta^{1} \\
u_{, 2}-\eta^{2} \\
\phi_{, 1} \\
\phi_{, 2} \\
-i \omega u
\end{array}\right)
$$

where $\mathrm{A}, \mathrm{B}$ and $\mathrm{C}$ are $2 \times 2$ matrices, $0_{n \times m}$ is a $n \times m$ zero matrix, and here and henceforth the assumed harmonic dependence on time is used to replace time derivatives with $-i \omega$. These quantities enter the the reduced form of the governing equations for antiplane shear waves:

$$
\begin{aligned}
\sigma_{, 1}^{13}\left(x_{1}, x_{2}, t\right)+\sigma_{, 2}^{23}\left(x_{1}, x_{2}, t\right)+f\left(x_{1}, x_{2}, t\right) & =-\rho\left(x_{1}, x_{2}\right) \omega^{2} u\left(x_{1}, x_{2}, t\right), \\
D_{, 1}^{1}\left(x_{1}, x_{2}, t\right)+D_{, 2}^{2}\left(x_{1}, x_{2}, t\right) & =q\left(x_{1}, x_{2}, t\right)
\end{aligned}
$$

The first step in the homogenization scheme is to rewrite Eq. (6) using the Bloch ansatz [Eq. (3)], which provides

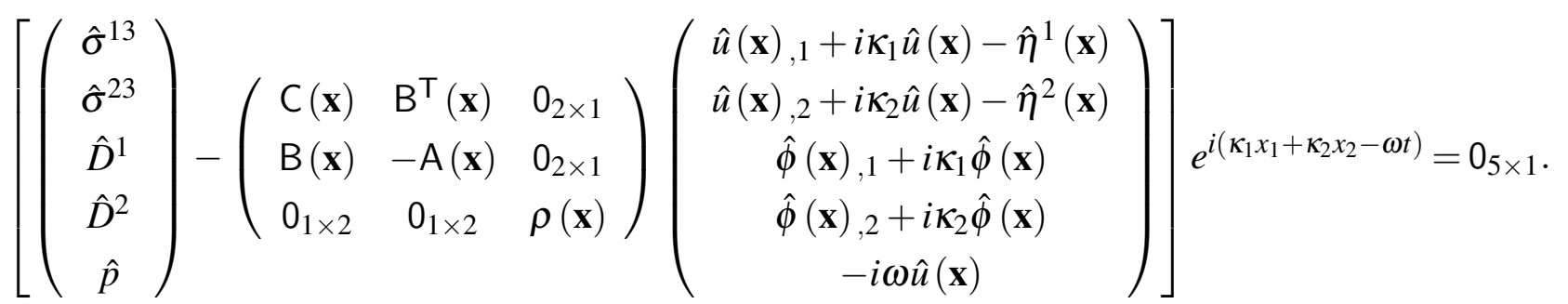

We expand the periodic parts in Eq. (8) in Fourier series:

$$
\hat{\zeta}(\mathbf{x})=\sum_{\mathbf{G}} \check{\zeta}_{\mathbf{G}} e^{i \mathbf{G} \cdot \mathbf{x}}, \check{\zeta}_{\mathbf{G}}:=|\Omega|^{-1} \int_{\Omega} \zeta(\mathbf{x}) e^{-i \mathbf{G} \cdot \mathbf{x}} \mathrm{d} \mathbf{x},
$$


where $\{\mathbf{G}\}$ is the infinite set of reciprocal lattice vectors; if we assume a square lattice with a period $a$, then $\left\{\mathbf{G}=\frac{2 \pi}{a} n_{1} \mathbf{e}_{1}+\frac{2 \pi}{a} n_{2} \mathbf{e}_{2}, n_{1}, n_{2} \in \mathbb{Z}\right\}$. The result reads

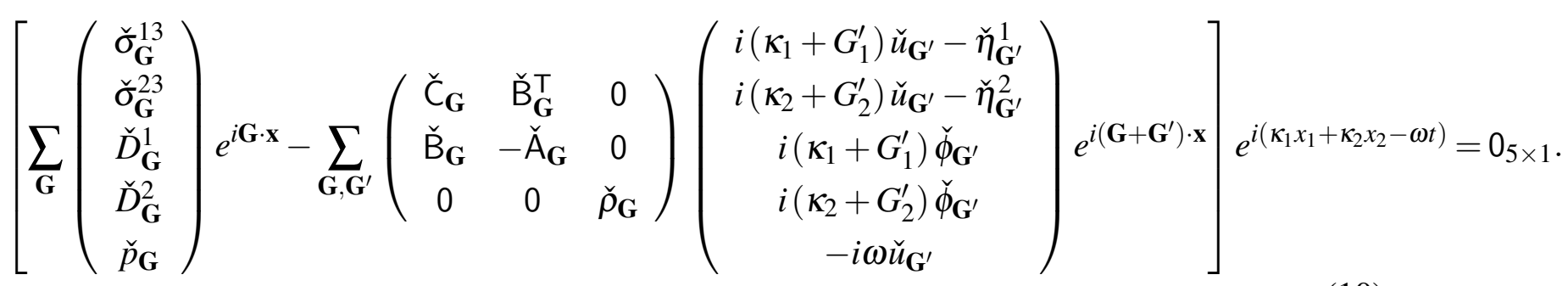

Since Eq. (10) holds for any value of $\mathbf{x}$ and $t$, the expression in the square brackets must vanish, which implies

$$
\sum_{\mathbf{G}}\left(\begin{array}{c}
\check{\sigma}_{\mathbf{G}}^{13} \\
\check{\sigma}_{\mathbf{G}}^{23} \\
\check{D}_{\mathbf{G}}^{1} \\
\check{D}_{\mathbf{G}}^{2} \\
\check{p}_{\mathbf{G}}
\end{array}\right) e^{i \mathbf{G} \cdot \mathbf{x}}=\sum_{\mathbf{G}, \mathbf{G}^{\prime}}\left(\begin{array}{ccc}
\check{C}_{\mathbf{G}} & \check{\mathrm{B}}_{\mathbf{G}}^{\top} & 0 \\
\check{\mathrm{B}}_{\mathbf{G}} & -\check{\mathrm{A}}_{\mathbf{G}} & 0 \\
0 & 0 & \check{\rho}_{\mathbf{G}}
\end{array}\right)\left(\begin{array}{c}
i\left(\kappa_{1}+G_{1}^{\prime}\right) \check{u}_{\mathbf{G}^{\prime}}-\check{\eta}_{\mathbf{G}^{\prime}}^{1} \\
i\left(\kappa_{2}+G_{2}^{\prime}\right) \check{u}_{\mathbf{G}^{\prime}}-\check{\eta}_{\mathbf{G}^{\prime}}^{2} \\
i\left(\kappa_{1}+G_{1}^{\prime}\right) \check{\phi}_{\mathbf{G}^{\prime}} \\
i\left(\kappa_{2}+G_{2}^{\prime}\right) \check{\phi}_{\mathbf{G}^{\prime}} \\
-i \omega \check{u}_{\mathbf{G}^{\prime}}
\end{array}\right) e^{i\left(\mathbf{G}+\mathbf{G}^{\prime}\right) \cdot \mathbf{x}}
$$

We multiply Eq. (10) by $\mathrm{e}^{-i \mathbf{G}^{\prime \prime} \cdot \mathbf{x}}$, and integrate the result over the unit cell. The orthogonality of the Fourier functions eliminates all terms, except those that satisfy $\mathbf{G}^{\prime \prime}=\mathbf{G}+\mathbf{G}^{\prime}$. Thus, the resultant equation (after changing $\mathbf{G}^{\prime \prime}$ to $\mathbf{G}$ ) is

$$
\left(\begin{array}{c}
\check{\sigma}_{\mathbf{G}}^{13} \\
\check{\sigma}_{\mathbf{G}}^{23} \\
\check{D}_{\mathbf{G}}^{1} \\
\check{D}_{\mathbf{G}}^{2} \\
\check{p}_{\mathbf{G}}^{2}
\end{array}\right)=\sum_{\mathbf{G}^{\prime}}\left(\begin{array}{ccc}
\check{\mathrm{C}}_{\mathbf{G G}^{\prime}} & \check{\mathrm{B}}_{\mathbf{G G}^{\prime}}^{\top} & 0 \\
\check{\mathrm{B}}_{\mathbf{G G}^{\prime}} & -\check{\mathrm{A}}_{\mathbf{G G}^{\prime}} & 0 \\
0 & 0 & \check{\rho}_{\mathbf{G G}^{\prime}}
\end{array}\right)\left(\begin{array}{c}
i\left(\kappa_{1}+G_{1}^{\prime}\right) \check{u}_{\mathbf{G}^{\prime}}-\check{\eta}_{\mathbf{G}^{\prime}}^{1} \\
i\left(\kappa_{2}+G_{2}^{\prime}\right) \check{u}_{\mathbf{G}^{\prime}}-\check{\eta}_{\mathbf{G}^{\prime}}^{2} \\
i\left(\kappa_{1}+G_{1}^{\prime}\right) \check{\phi}_{\mathbf{G}^{\prime}} \\
i\left(\kappa_{2}+G_{2}^{\prime}\right) \check{\phi}_{\mathbf{G}^{\prime}} \\
-i \omega \check{u}_{\mathbf{G}^{\prime}}
\end{array}\right)
$$

where $(\cdot)_{\mathbf{G G}^{\prime}}$ denotes the Fourier coefficient of $(\cdot)$ along the basis function $\mathrm{e}^{i\left(\mathbf{G}-\mathbf{G}^{\prime}\right) \cdot \mathbf{x}}$.

Having the constitutive equations in the desired form, we proceed to manipulate the governing equations in the same manner. Accordingly, we consider the Bloch form of Eq. (7), namely,

$$
\begin{aligned}
& \left(\hat{\sigma}^{13}{ }_{, 1}+i \kappa_{1} \hat{\sigma}^{13}+\hat{\sigma}^{23}{ }_{, 2}+i \kappa_{2} \hat{\sigma}^{23}+\hat{f}+i \omega \hat{p}\right) e^{i(\boldsymbol{\kappa} \cdot \mathbf{x}-\omega t)}=0, \\
& \left(\hat{D}_{, 1}^{1}+i \kappa_{1} \hat{D}^{1}+\hat{D}_{, 2}^{2}+i \kappa_{2} \hat{D}^{2}-\hat{q}\right) e^{i(\boldsymbol{\kappa} \cdot \mathbf{x}-\omega t)}=0 .
\end{aligned}
$$

Again, since Eq. (13) holds for any value of $\mathbf{x}$ and $t$, the expressions in the brackets must vanish, 
which allows us to obtain

$$
\begin{aligned}
& \sum_{\mathbf{G}}\left[i\left(\kappa_{1}+G_{1}\right) \check{\sigma}_{\mathbf{G}}^{13}+i\left(\kappa_{2}+G_{2}\right) \check{\sigma}_{\mathbf{G}}^{23}+\check{f}_{G}\right] e^{i \mathbf{G} \cdot \mathbf{x}}=-i \omega \sum_{\mathbf{G}} \check{p}_{\mathbf{G}} e^{i \mathbf{G} \cdot \mathbf{x}}, \\
& \sum_{\mathbf{G}}\left[i\left(\kappa_{1}+G_{1}\right) \check{D}_{\mathbf{G}}^{1}+i\left(\kappa_{2}+G_{2}\right) \check{D}_{\mathbf{G}}^{2}\right] e^{i \mathbf{G} \cdot \mathbf{x}}=\sum_{\mathbf{G}} \check{q}_{\mathbf{G}} e^{i \mathbf{G} \cdot \mathbf{x}}
\end{aligned}
$$

after expanding the periodic parts in Fourier series. We multiply Eq. (14) by $e^{-i \mathbf{G}^{\prime \prime} \cdot \mathbf{x}}$, and integrate the result over the unit-cell. This provides for each $\mathbf{G}$

$$
\mathrm{D}_{\mathbf{G}}^{\top} \mathrm{h}_{\mathbf{G}}=-\mathrm{f}_{\mathbf{G}}
$$

where

$$
\mathrm{D}_{\mathbf{G}}=\left(\begin{array}{cc}
i\left(\kappa_{1}+G_{1}\right) & 0 \\
i\left(\kappa_{2}+G_{2}\right) & 0 \\
0 & \left(\kappa_{1}+G_{1}\right) \\
0 & i\left(\kappa_{2}+G_{2}\right) \\
i \omega & 0
\end{array}\right), \mathrm{h}_{\mathbf{G}}=\left(\begin{array}{c}
\check{\sigma}_{\mathbf{G}}^{13} \\
\check{\sigma}_{\mathbf{G}}^{23} \\
\check{D}_{\mathbf{G}}^{1} \\
\check{D}_{\mathbf{G}}^{2} \\
\check{p}_{\mathbf{G}}
\end{array}\right), \mathrm{f}_{\mathbf{G}}=\left(\begin{array}{c}
\check{f}_{\mathbf{G}} \\
-\check{q}_{\mathbf{G}}
\end{array}\right)
$$

Next, we assemble all the matrix equations associated with Eq. (15) for each $\mathbf{G}$ to a single infinite matrix system in the form

$$
\mathrm{D}_{\mathrm{A}}^{\top} \mathrm{h}_{\mathrm{A}}=-\mathrm{f}_{\mathrm{A}} .
$$

For computational purpose, the size of the matrices and column vectors in Eq. (17) is truncated by defining the number of Fourier components (equivalently, the number of $\mathbf{G}$ vectors) to $N$. In this case, $\mathrm{f}_{\mathrm{A}}$ is a column vector of $2 N$ components which contains all the Fourier coefficients of $\check{f}_{\mathbf{G}}$ and $\check{q}_{\mathbf{G}}, \mathrm{h}_{\mathrm{A}}$ is a column vector of $5 N$ components which contains all the Fourier coefficients of $\check{\sigma}_{\mathbf{G}}^{13}, \check{\sigma}_{\mathbf{G}}^{23}, \check{D}_{\mathbf{G}}^{1}, \check{D}_{\mathbf{G}}^{2}$ and $\check{p}_{\mathbf{G}}$, and $\mathrm{D}_{\mathrm{A}}$ is a $5 N \times 2 N$ matrix, the components of which are given in Appendix A. The column vector $h_{A}$ is obtained in the same manner, by assembling the matrix equations associated with Eq. (12), such that

$$
\mathrm{h}_{\mathrm{A}}=\mathrm{L}_{\mathrm{A}}\left(\mathrm{J}_{\mathrm{A}} \mathrm{w}_{\mathrm{A}}-\mathrm{m}_{\mathrm{A}}\right)
$$

where $\mathrm{L}_{\mathrm{A}}$ is a $5 N \times 5 N$ matrix that assembles the Fourier components of the mechanical properties, $\mathrm{J}_{\mathrm{A}}$ is a $5 N \times 2 N$ matrix that assembles the Fourier components of the differential operators, $\mathrm{w}_{\mathrm{A}}$ is a column vector of $2 \mathrm{~N}$ components that assembles the Fourier components of the displacement and the electric potential and $\mathrm{m}_{\mathrm{A}}$ is a column vector of $5 \mathrm{~N}$ components that assembles the Fourier components of the inelastic strain. Explicit expressions for Eqs. (17) and (18) are given in Appendix A. We now extract from Eq. (18) the average fields out of $h_{A}$, and write them as 


$$
\overline{\mathrm{h}}:=\left(\begin{array}{c}
\bar{\sigma}^{13} \\
\bar{\sigma}^{23} \\
\bar{D}^{1} \\
\bar{D}^{2} \\
\bar{p}
\end{array}\right) \equiv\left(\begin{array}{c}
\check{\sigma}_{0}^{13} \\
\check{\sigma}_{0}^{23} \\
\check{D}_{0}^{1} \\
\check{D}_{0}^{2} \\
\check{p}_{0}
\end{array}\right)=\mathrm{L}_{0}\left\{\mathrm{~J}_{0} \overline{\mathrm{w}}-\overline{\mathrm{m}}\right\}+\mathrm{L}_{s} \mathrm{~J}_{s} \mathrm{w}_{s},
$$

where $J_{0}$ and $L_{0}$ are the parts of the matrices that multiply the average fields (associated with $\mathbf{G}=\mathbf{0}$ ) and $(\circ)_{s}$ denotes the reduced matrix or vector without the $\mathbf{G}=\mathbf{0}$ terms ${ }^{4}$. Our goal is to express the terms $w_{s}$ using $\bar{w}$ and $\bar{m}$. To this end, we use Eq. (17) to obtain

$$
D_{A}^{T} L_{A}\left(J_{A} w_{A}-m_{A}\right)=-f_{A}
$$

Next, we utilize the equations that do not include $\mathbf{G}=\mathbf{0}$ in Eq. (20) to write

$$
\mathrm{Q}_{s} \mathrm{w}_{s}=-\mathrm{T}\left\{\mathrm{J}_{0} \overline{\mathrm{w}}-\overline{\mathrm{m}}\right\}
$$

where $\mathrm{Q}_{s}$ is a square matrix which contain the terms which do not multiply the average fields and $T$ is the part of the matrix $D_{A}^{T} L_{A}$ which multiplies the average fields. (This process is similar to the one carried out in Ref. [48] for flexural waves in Euler-Bernoulli beams.) We are now able to express the fluctuating terms of $w$ using its average value and the properties of the composite, namely,

$$
\mathrm{w}_{s}=-\mathrm{Q}_{s}^{-1} \mathrm{~T}\left\{\mathrm{~J}_{0} \overline{\mathrm{w}}-\overline{\mathrm{m}}\right\}
$$

Accordingly, Eq. (19) reads

$$
\overline{\mathrm{h}}=\left(\mathrm{L}_{0}-\mathrm{L}_{s} \mathrm{~J}_{s} \mathrm{Q}_{s}^{-1} \mathrm{~T}\right)\left\{\mathrm{J}_{0} \overline{\mathrm{w}}-\overline{\mathrm{m}}\right\}
$$

Eq. (23) is the desired form, since it relates the independent kinematic variables $J_{0} \bar{w}$ to the dependent kinetic variables $\bar{h}$ through a matrix that defines the effective properties. These are given through the following identification

$$
\left(\begin{array}{ccc}
\tilde{\mathrm{C}} & \tilde{\mathrm{B}}^{\dagger} & \tilde{\mathrm{S}} \\
\tilde{\mathrm{B}} & -\tilde{\mathrm{A}} & \tilde{\mathrm{W}} \\
\tilde{\mathrm{S}}^{\dagger} & \tilde{\mathrm{W}}^{\dagger} & \tilde{\rho}
\end{array}\right):=\mathrm{L}_{0}-\mathrm{L}_{s} \mathrm{~J}_{s} \mathrm{Q}_{s}^{-1} \mathrm{~T}
$$

The resultant effective properties are nonlocal in time and space, since they are functions of $\omega$ and $\boldsymbol{\kappa}$. In general, $\boldsymbol{\omega}$ and $\boldsymbol{\kappa}$ are independent and prescribed by the impressed sources; only if the sources

\footnotetext{
${ }^{4}$ Here $\mathrm{L}_{0}$ is a $5 \times 5$ matrix, $\mathrm{J}_{0}$ is a $5 \times 2$ matrix, $\overline{\mathrm{w}}$ is a vector with 2 components, $\overline{\mathrm{m}}$ is a vector with 5 components, $\mathrm{L}_{s}$ is a $5 \times(5 N-5)$ matrix, $\mathrm{J}_{s}$ is a $(5 N-5) \times(2 N-2)$ matrix and $\mathrm{w}_{s}$ is a vector with $2 N-2$ components.
} 
are set to zero, then $(\omega, \boldsymbol{\kappa})$ pairs are related through the dispersion relations that are defined by the normal modes of the composites. Notably, the nonzero blocks $\tilde{W}$ and $\tilde{W}^{\dagger}$ are identified with the electromomentum couplings that were first discovered by Pernas-Salomón and Shmuel [49], using

a different formulation that is based on Green's functions. Like $\tilde{S}$, the electromomentum coupling is of Willis type, in the sense that it couples fields that are not coupled at the microscopic level, and arises at finite frequencies and/or wavevectors. Symbolically, we write the effective constitutive relations as

$$
\left(\begin{array}{c}
\langle\boldsymbol{\sigma}\rangle \\
\langle\mathbf{D}\rangle \\
\langle\mathbf{p}\rangle
\end{array}\right)=\left(\begin{array}{ccc}
\tilde{\mathbf{C}} & \tilde{\mathbf{B}}^{\dagger} & \tilde{\mathbf{S}} \\
\tilde{\mathbf{B}} & -\tilde{\mathbf{A}} & \tilde{\mathbf{W}} \\
\tilde{\mathbf{S}}^{\dagger} & \tilde{\mathbf{W}}^{\dagger} & \tilde{\boldsymbol{\rho}}
\end{array}\right)\left(\begin{array}{c}
\langle\nabla \mathbf{u}\rangle-\boldsymbol{\eta} \\
\langle\nabla \phi\rangle \\
\langle\dot{\mathbf{u}}\rangle
\end{array}\right)
$$

Refs. [41, 45, 50, 57] discuss how the nonlocal nature of the effective operator generates an ambiguity in the definition of the independent effective variables. They suggest a more physical set by adding the time rate of the original independent effective variables. We adapt this notion, and define an alternative form to Eq. (25), namely,

$$
\left(\begin{array}{c}
\langle\boldsymbol{\sigma}\rangle \\
\langle\mathbf{D}\rangle \\
\langle\mathbf{p}\rangle
\end{array}\right)=\left(\begin{array}{ccc}
\tilde{\mathbf{C}} & \tilde{\mathbf{B}}^{\dagger} & \mathbf{0} \\
\tilde{\mathbf{B}} & -\tilde{\mathbf{A}} & \mathbf{0} \\
\mathbf{0} & \mathbf{0} & \tilde{\boldsymbol{\rho}}
\end{array}\right)\left(\begin{array}{c}
\langle\nabla \mathbf{u}\rangle-\boldsymbol{\eta} \\
\langle\nabla \phi\rangle \\
\langle\dot{\mathbf{u}}\rangle
\end{array}\right)+\left(\begin{array}{ccc}
\mathbf{0} & \mathbf{0} & \widehat{\mathbf{S}} \\
\mathbf{0} & \mathbf{0} & \widehat{\mathbf{W}} \\
\widehat{\mathbf{S}}^{\dagger} & \widehat{\mathbf{W}}^{\dagger} & \mathbf{0}
\end{array}\right)\left(\begin{array}{c}
\langle\nabla \dot{\mathbf{u}}\rangle-\dot{\boldsymbol{\eta}} \\
\langle\nabla \dot{\boldsymbol{\phi}}\rangle \\
\langle\ddot{\mathbf{u}}\rangle
\end{array}\right),
$$

where $\widehat{\mathbf{S}}=i \tilde{\mathbf{S}} / \omega, \widehat{\mathbf{W}}=i \tilde{\mathbf{W}} / \omega$ and so on. Accordingly, the modified Willis coupling actually depends on the acceleration rather than on the velocity. Likewise, the electromomentum coupling actually depends on the time rate of the electric field.

\section{Numerical analysis}

In this section, we apply the method that we have developed in Sec. 3 to numerically evaluate the effective properties - and specifically the electromomentum tensor-of exemplary piezoelectric composites. Specifically, we consider two compositions of PZT4 fibers within a PMMA matrix, the properties of which are given in Tab. 1. The phases are poled in the $x_{3}$-direction, such that in the $x_{1}-x_{2}$ plane they exhibit transverse isotropy. We consider a square lattice with the period $a=5 \mathrm{~mm}$, and analyze two different cross-sections: one with a circular fiber of a radius $R=1.9 \mathrm{~mm}$ (Fig. 1a), and one with a circular sector that is defined by the area $\pi R^{2} / 8$ (Fig. 1b). Before we present our results, we note that our scheme was validated against several benchmark cases, as detailed in appendix B. In Sec. 4.1, we show that the effective model defined by our homogenization scheme also exhibits the same dispersion relation as the composite in the case of free waves. As 


\begin{tabular}{ccccc}
\hline Material properties & $\rho\left[\frac{\mathrm{kg}}{\mathrm{m}^{3}}\right]$ & $A\left[\frac{\mathrm{nF}}{\mathrm{m}}\right]$ & $B\left[\frac{\mathrm{C}}{\mathrm{m}^{2}}\right]$ & $C[\mathrm{GPa}]$ \\
\hline \hline PMMA & 1188 & 0.023 & 0 & 3.3 \\
\hline PZT4 & 7500 & 5.6 & 15.1 & 115 \\
\hline
\end{tabular}

Table 1: The mass density, dielectric, piezoelectric and elastic moduli of PMMA and PZT4. These materials are used in the computations as the matrix and fiber phases, respectively, of a composite with square lattice of period $a=5 \mathrm{~mm}$. We consider two different PZT4 fibers: one with a circular cross-section of a radius $R=1.9 \mathrm{~mm}$, and one with a circular sector that is defined by the area $\pi R^{2} / 8$.

(a)

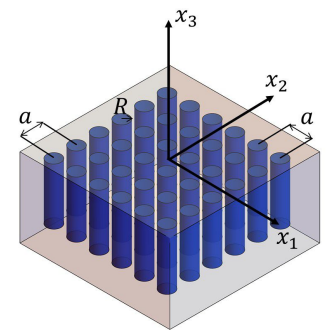

(b)

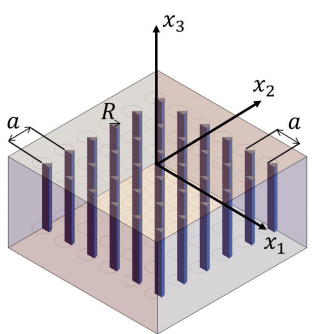

(c)

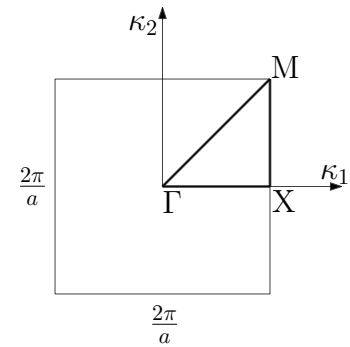

Fig. 1: Illustration of the piezoelectric composites with (a) circular; and (b) circular sector fibers in a square lattice of period $a$. (c) The points $\mathrm{X}, \Gamma$ and $\mathrm{M}$ in the corresponding $1^{\text {st }}$ Brillouin zone.

per our discussion in Sec. 1, this reinforcing feature on its own is not sufficient to validate the model, since the effective properties should also satisfy the mathematical restrictions imposed by causality, energy conservation and reciprocity, as we demonstrate in the sequel. By contrast, we will also show that homogenized models that ignore the electromomentum tensor violate these physical laws, even if these models recover the dispersion relation of the composite.

\subsection{Free waves}

We begin by analyzing the effective properties in the case of free waves, i.e., in the absence of sources, where we recall that the effective properties in such case are not uniquely defined. It is important to note that nonlocality induces components in the constitutive tensor that do not appear in local constitutive equations, even if the medium is isotropic, see appendix C. Thus, to avoid information overload, we restrict attention to selected components of the effective tensors, and to the section $-X-\Gamma-X$ of the $1^{\text {st }}$ Brillouin zone (Fig. 1c).

We begin by presenting in Fig. 2 the effective properties of the axisymmetric unit cell as functions of normalized frequency $\hat{\omega}=\omega a / 2 \pi c_{m}$, where $c_{m}$ is the velocity of shear wave in the PMMA matrix. We observe that all the standard properties- $\tilde{\rho}, \tilde{\mathbf{A}}, \tilde{\mathbf{B}}$ and $\tilde{\mathbf{C}}$-are real and satisfy the reci- 
procity requirement $[50]$

$$
\tilde{\rho}(\boldsymbol{\kappa})=\tilde{\rho}(-\boldsymbol{\kappa}), \tilde{A}_{i j}(\boldsymbol{\kappa})=\tilde{A}_{j i}(-\boldsymbol{\kappa}), \tilde{B}_{J i}^{\dagger}(\boldsymbol{\kappa})=\tilde{B}_{i J}(-\boldsymbol{\kappa}), \tilde{C}_{I J}(\boldsymbol{\kappa})=\tilde{C}_{J I}(-\boldsymbol{\kappa}) ;
$$

here and henceforth uppercase indices run from 1 to 6 according to Voigt notation. (For example, the components $\tilde{B}_{113}, \tilde{C}_{2323}$ and $\widehat{S}_{331}^{\dagger}$ are denoted $\tilde{B}_{15}, \tilde{C}_{44}$ and $\widehat{S}_{35}$, respectively.)

We examine next the coupling of Willis type $\widehat{\mathbf{S}}$ and $\widehat{\mathbf{W}}$ (and their adjoints), the computation of which demonstrates the following features, supporting the validity of our homogenization scheme. (i) The tensors $\widehat{\mathbf{S}}, \widehat{\mathbf{S}}^{\dagger}, \widehat{\mathbf{W}}$ and $\widehat{\mathbf{W}}^{\dagger}$ are zero at $\boldsymbol{\kappa}=\mathbf{0}$, and pure imaginary for $\boldsymbol{\kappa} \neq \mathbf{0}$, in agreement with the understanding that the imaginary part captures nonlocal interactions, and that the real part should vanish when there is inversion symmetry ${ }^{5}$ [49, 57]; (ii) the tensors satisfy the symmetries

$$
\widehat{S}_{53}^{*}(\boldsymbol{\kappa})=\widehat{S}_{35}^{\dagger}(\boldsymbol{\kappa})=\widehat{S}_{53}(-\boldsymbol{\kappa}), \widehat{W}_{13}^{*}(\boldsymbol{\kappa})=\widehat{W}_{31}^{\dagger}(\boldsymbol{\kappa})=\widehat{W}_{13}(-\boldsymbol{\kappa}),
$$

as required from reciprocity and energy conservation [50].

From the calculation of the effective properties, it is possible to evaluate the dispersion relation that the homogenized medium exhibits. To do so, we substitute the effective constitutive relations [Eq. (26)] into the macroscopic governing equations [as mentioned, they are of the same form of Eq. (1), where the effective fields replace the microscopic ones], and extract $\omega(\boldsymbol{\kappa})$ relation after some algebraic manipulation, as detailed in appendix D. This resultant dispersion relation along the points ГХМГ is shown in Fig. 2g, where we compare the corresponding acoustic and optical branches of the homogeneous medium (green circle marks), with the branches of the composite (black solid curves). Remarkably, the prediction of the homogenized medium recover exactly the curves of the composite.

We recall that there is an ambiguity in the definition of the Bloch vector: if $\boldsymbol{\kappa}$ is a solution, then so is the sum of $\boldsymbol{\kappa}$ with any reciprocal lattice vector. To avoid this ambiguity, $\boldsymbol{\kappa}$ is restricted to the $1^{\text {st }}$ Brillouin zone. Willis [76] questioned if this ambiguity extends to the definition of the effective properties, when later Srivastava and Nemat-Nasser [63] and Pernas-Salomón and Shmuel [48] showed that homogenizing only over the $1^{\text {st }}$ Brillouin zone leads to nonphysical results. Specifically, Pernas-Salomón and Shmuel [48], who developed a similar method to calculate the effective properties of a periodic Euler-Bernoulli beam, showed that the effective properties using values of $\boldsymbol{\kappa}$ from the $1^{\text {st }}$ Brillouin zone do not recover branches in the dispersion relation beyond the acoustic branch $^{6}$, unless the values of $\boldsymbol{\kappa}$ are taken from ascending Brillouin zones. They further showed

\footnotetext{
${ }^{5}$ Of course, the role of the imaginary and real part of the cross-couplings in their standard form [Eq. (25)] is exchanged.

${ }^{6}$ Norris et al. [47], for example, used a similar homogenization method to calculate the effective properties and demonstrated them as function of the wave-number. With these results, one cannot recover the dispersion relation from the second branch and above it.
} 
(a)

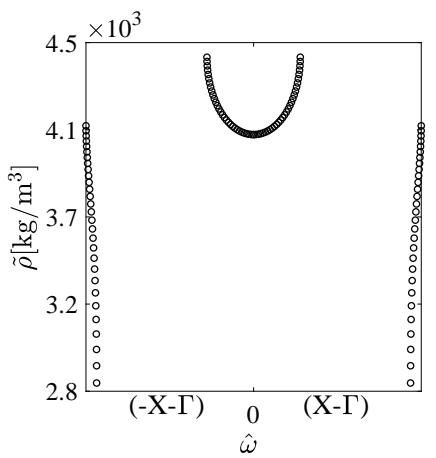

(d)

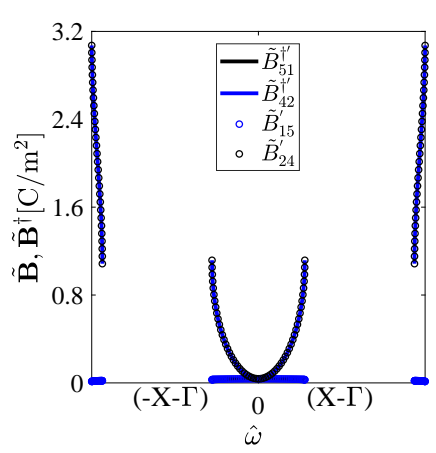

(b)

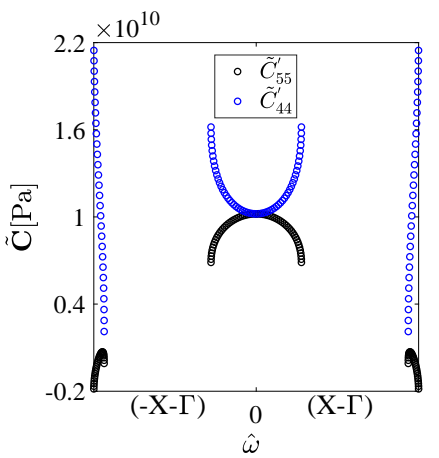

(e)

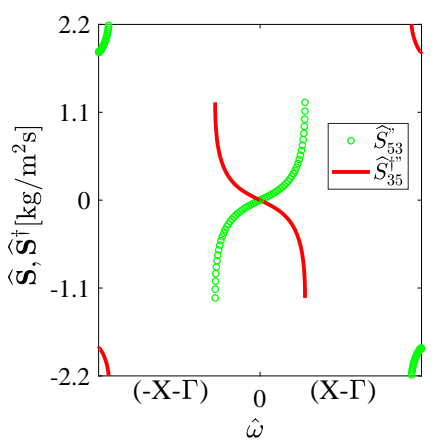

(g)

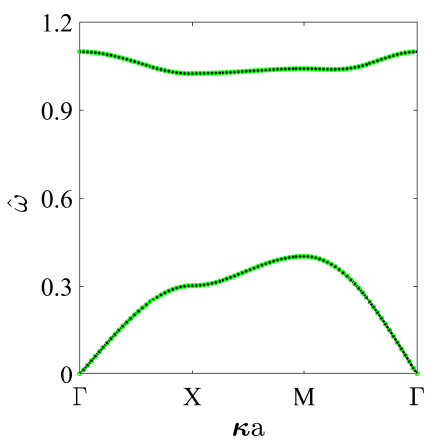

(c)

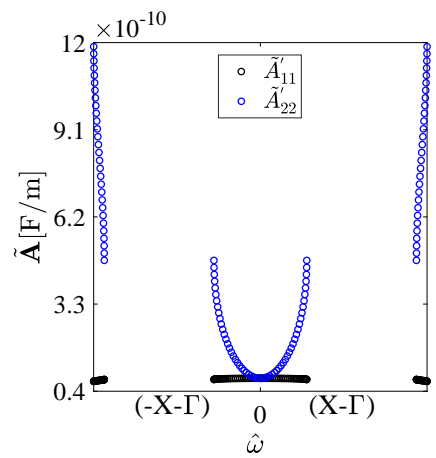

(f)

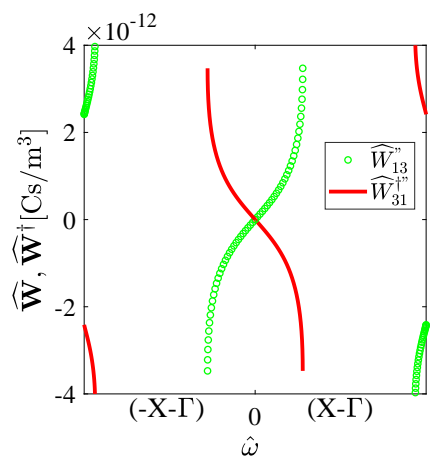

Fig. 2: Effective properties as functions of the normalized frequency that is associated with the wavevector along $-\mathrm{X}-\Gamma-\mathrm{X}$, for PZT4 circular fibers in a PMMA matrix, the properties of which are given in Tab. 1. (a) Mass density; and selected components of the (b) elastic; (c) dielectric; (d) piezoelectric; (e) Willis; and (f) electromomentum tensors. (g) Resultant acoustic and optical branches of the dispersion relations of the homogenized model along the points ГХМГ (green circles). The two branches of the original composite are shown in solid black curves. 
that these properties lead to unphysical reflections in scattering problems, see also Ref. [63]. This holds here as well: values of $\boldsymbol{\kappa}$ in the $1^{\text {st }}$ Brillouin zone do not recover the optical branch of the original composite. Accordingly, to recover the second branch we have selected values of $\boldsymbol{\kappa}$ from the second Brillouin zone.

Fig. 3 presents the effective properties of the asymmetric unit cell. Again, we restrict attention to the section $-\mathrm{X}-\Gamma-\mathrm{X}$ of the Brillouin zone and to selected components of the effective tensors. The observations regarding the standard properties that were noted for the axisymmetric cell apply also here: the properties are real and satisfy the reciprocity requirement [Eq. (27)]. In contrast, the computation of the couplings of Willis type $\widehat{\mathbf{S}}, \widehat{\mathbf{W}}$ and their adjoints provides different results than those of the axisymmetric cell, namely, here they do not vanish at $\boldsymbol{\kappa}=\mathbf{0}$. Specifically, they are pure real in this limit, and become complex-valued for finite $\boldsymbol{\kappa}$. Furthermore, we observe that their real part is even in $\boldsymbol{\kappa}$, in consistency with reciprocity [50]. The evaluation of the dispersion relation using the homogenized properties is carried out in Fig. 3i, and is compared with the dispersion relation of the original composite. Here again, the dispersion relation that the effective medium predicts recovers exactly the dispersion relation of the composite.

As mentioned, since there are no sources, the definition of the effective properties is not unique, and it is possible to define equivalent properties that neglect $\mathbf{W}$ (and $\mathbf{W}^{\dagger}$ ), while recovering the dispersion relation. However, we show next that equivalent properties without the electromomentum coupling are not physical, since they violate reciprocity and energy conservation. To do so, we note first that owing to the absence of sources, the independent fields are in fact algebraically dependent, as known from studies on standard (i.e., purely elastic) Willis materials [45]. Here, they are related via (appendix D)

$$
\langle\nabla u\rangle=-\frac{\kappa}{\omega}\langle\dot{u}\rangle,\langle\dot{u}\rangle=-\frac{\kappa^{\top}\langle\nabla u\rangle}{\kappa^{\top} \kappa} \omega,\langle\dot{u}\rangle=\frac{\kappa^{\top} \tilde{A}}{-i \omega \kappa^{\top} \widehat{W}-\omega^{-1} \kappa^{\top} \tilde{B} \kappa}\langle\nabla \phi\rangle,
$$

for a generalized Willis medium that exhibits the piezoelectric and electromomentum effects. Accordingly, it is possible to absorb $\widehat{W}$ into an equivalent dielectric tensor:

$$
\mathrm{A}_{\mathrm{eq}}=\tilde{\mathrm{A}}+i \omega \widehat{\mathrm{W}} \frac{\kappa^{\top} \tilde{\mathrm{A}}}{-i \omega \kappa^{\top} \widehat{\mathrm{W}}-\omega^{-1} \kappa^{\top} \tilde{\mathrm{B}} \kappa},
$$

that define the following equivalent constitutive equation for the electric displacement field:

$$
\langle\mathrm{D}\rangle=\mathrm{B}_{\mathrm{eq}}\langle\nabla u\rangle-\mathrm{A}_{\mathrm{eq}}\langle\nabla \phi\rangle, \mathrm{B}_{\mathrm{eq}}=\tilde{\mathrm{B}}
$$

By construction, the equivalent description recovers the dispersion relation without using $\widehat{\mathbf{W}}$, however it violates the energy conservation and reciprocity conditions ${ }^{7} \tilde{A}_{i i}(\boldsymbol{\kappa})=\tilde{A}_{i i}^{*}(\boldsymbol{\kappa})=\tilde{A}_{i i}(-\boldsymbol{\kappa})$ (no

\footnotetext{
${ }^{7}$ These conditions in electrodynamics can be found in Ref. [25], Eq. (101.2) and Chapt. XII therein; their applica-
} 
(a)

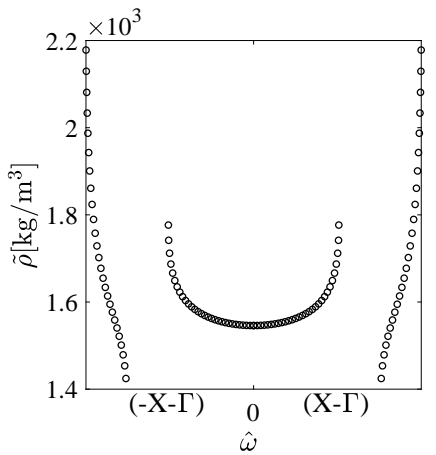

(d)

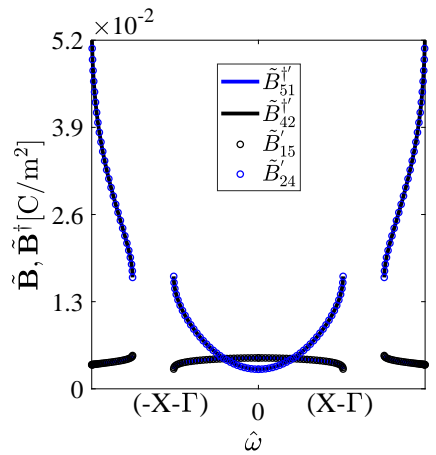

(g)

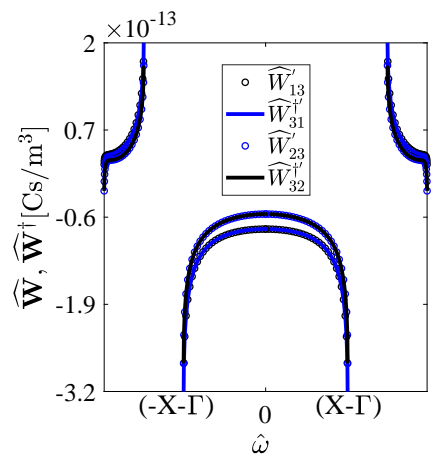

(b)

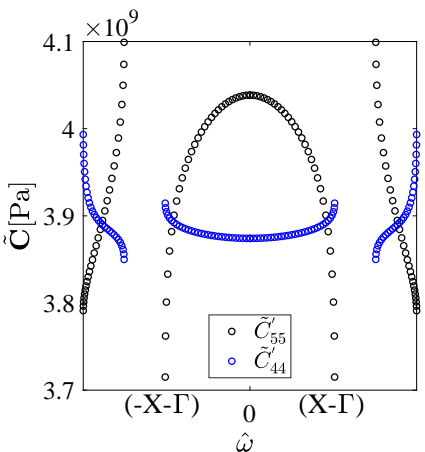

(e)

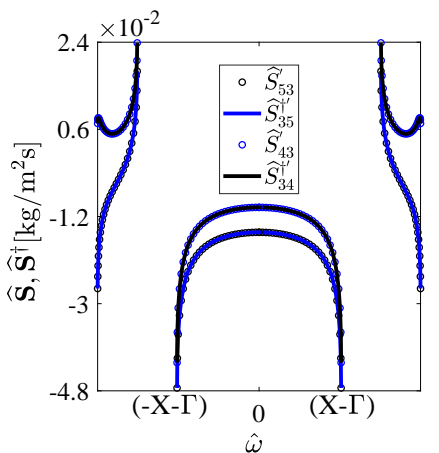

(h)

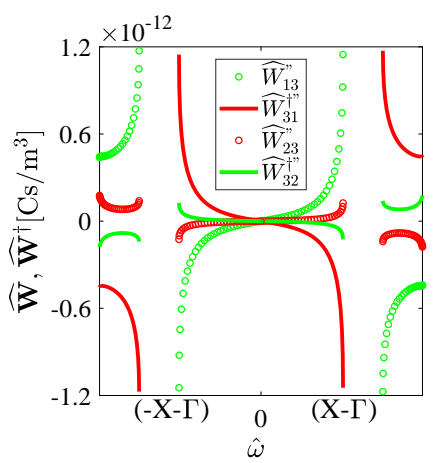

(c)

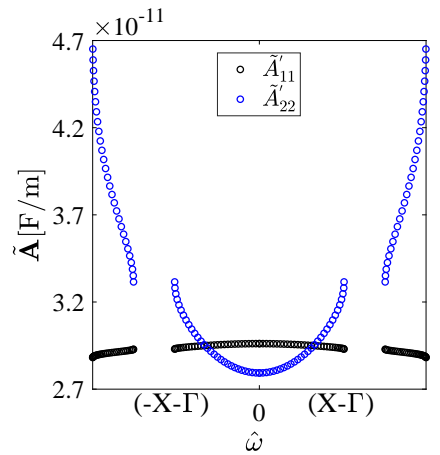

(f)

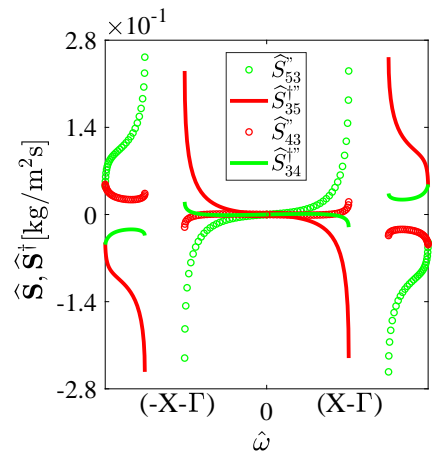

(i)

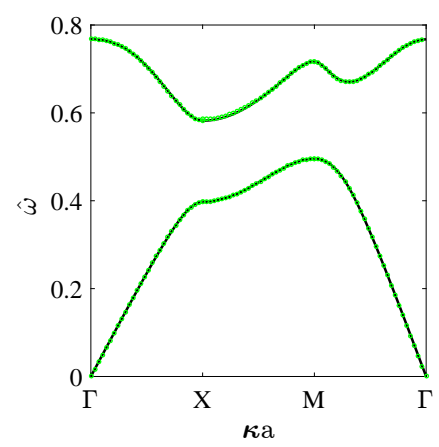

Fig. 3: Effective properties as functions of the normalized frequency that is associated with the wavevector along $-\mathrm{X}-\Gamma-\mathrm{X}$, for PZT4 circular sector fibers in a PMMA matrix, the properties of which are given in Tab. 1. (a) Mass density and selected components of the (b) elastic; (c) dielectric; (d) and piezoelectric tensors. Panels e and f (resp. $\mathrm{g}$ and $\mathrm{h}$ ) show the real and imaginary parts of the Willis tensor (resp. the electromomentum tensor). (i) Resultant acoustic and optical branches of the dispersion relations of the homogenized model along the points ГХМГ (green circles). The two branches of the original composite are shown in solid black curves. 
(a)

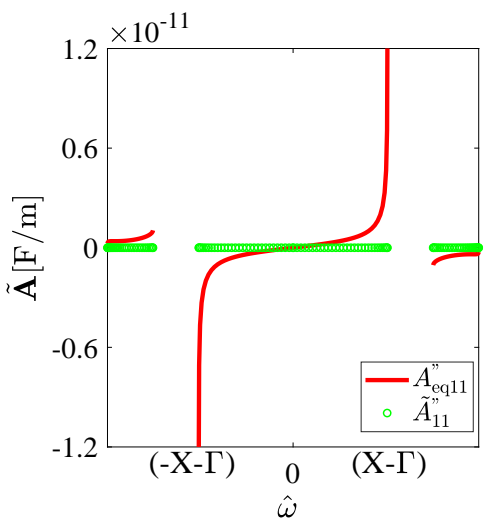

(b)

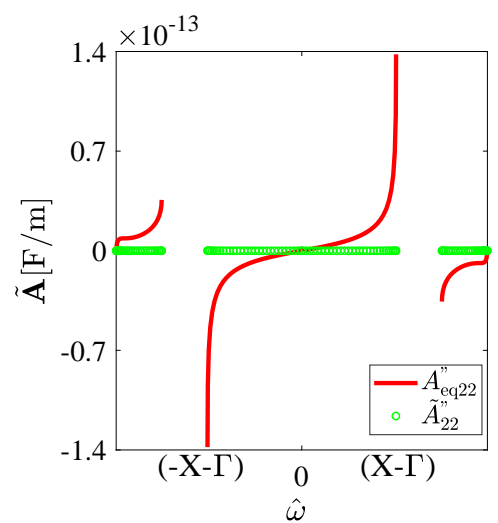

Fig. 4: The imaginary part of the diagonal components of the effective (green circles) and equivalent (red curves) dielectric tensor, calculated for the composite with asymmetric fibers. The equivalent imaginary components are nonzero and odd in $\boldsymbol{\kappa}$, hence violate energy conservation and reciprocity.

sum over $i$ ). This violation can be identified immediately from Eq. (30), by inspecting the symmetries that its comprising components $(\widehat{\mathrm{W}}, \tilde{\mathrm{A}}$ and $\tilde{B})$ satisfy, and deducing that the diagonal terms of $\tilde{A}_{\text {eq }}$ have a nonzero imaginary part that is odd $\boldsymbol{\kappa}$. This is demonstrated Fig. 4, where A" (red curves) that is extracted for the composite with the asymmetric fibers is presented as function of the frequency of the dispersion curves along $-X-\Gamma-X$. For comparison, we also present $\tilde{A}^{\prime \prime}$ (green circles); it is identically zero, as is should be.

\subsection{Local approximation of the effective relations}

The most prevalent use of metamaterials is in the long-wavelength regime, where the generated fields have a length much larger than the period of the composite. In such cases, the response of a metamaterial can be approximated as local, which implies that in our scheme we can approximate $\boldsymbol{\kappa}+\mathbf{G}$ by $\mathbf{G}$. We analyze next the local effective properties that result from this approximation. For brevity, we focus on the richer case of asymmetric fibers and present only selected components of the effective properties, where for completeness additional components are given in Appendix E. The selected properties as functions of normalized frequency are displayed in Fig. 5, and exhibit the following notable features. First, they respect causality, passivity and energy conservation, since they satisfy ${ }^{8}[4,57]$

$$
\frac{\partial \rho}{\partial \omega} \geq 0, \frac{\partial \tilde{C}_{I J}}{\partial \omega} \leq 0, \frac{\partial \tilde{A}_{i j}}{\partial \omega} \geq 0
$$

bility to generalized Willis media was shown by Pernas-Salomón and Shmuel [50].

${ }^{8}$ We note that the derivatives in Eq. (32) should be evaluated at a fixed $\boldsymbol{\kappa}$, as the partial derivates symbol implies. This requirement does not hold for the total derivatives, which correspond to the slopes in Figs. 2-3. (There, the properties are evaluated as functions of the frequency on the dispersion curves, where it is a function of $\boldsymbol{\kappa}$.) 
(a)

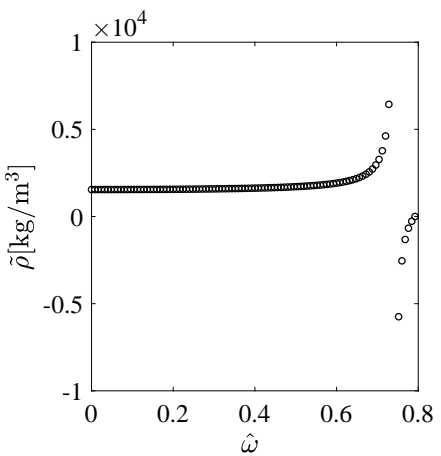

(d)

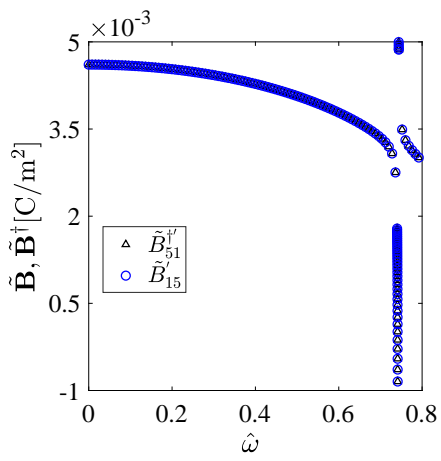

(b)

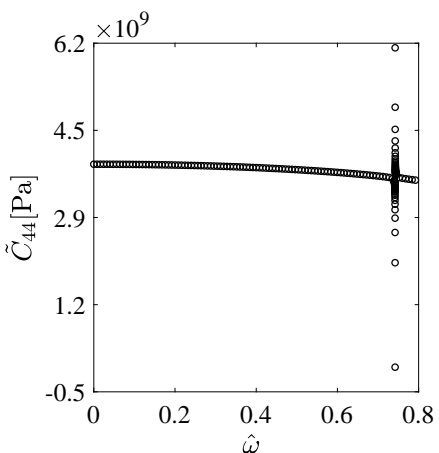

(e)

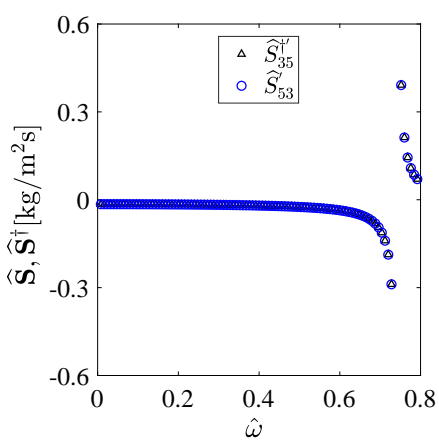

(c)

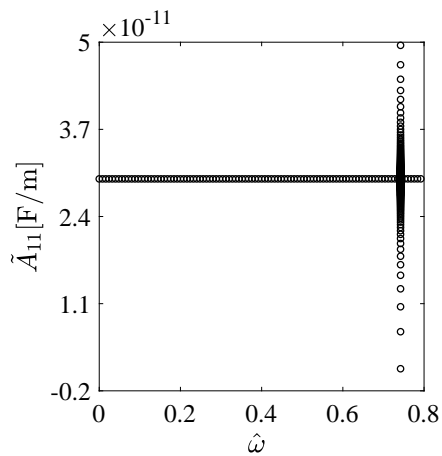

(f)

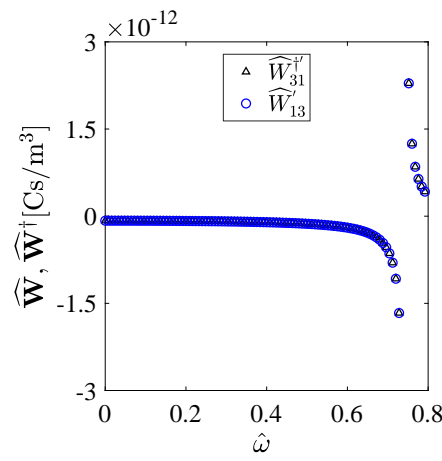

Fig. 5: The local approximation ( $\boldsymbol{\kappa}=\mathbf{0}$ ) of the effective (a) mass density; and selected components of the (b) elastic; (c) dielectric; (d) piezoelectric; (e) Willis; and (f) electromomentum tensors as functions of the normalized frequency, calculated for PZT4 circular sector fibers in a PMMA matrix (Tab. 1).

and they are all real. They exhibit a resonance at the normalized frequency $\approx 0.74$, where beyond this frequency they change sign. Furthermore, we observe that the cross couplings of Willis type also respect causality, passivity and energy conservation since they satisfy [50]

$$
\frac{\partial \tilde{B}_{i J}}{\partial \omega} \leq 0, \frac{\partial \widehat{S}_{I j}}{\partial \omega} \leq 0, \frac{\partial \widehat{W}_{i j}}{\partial \omega} \leq 0, \frac{\partial \tilde{B}_{I j}^{\dagger}}{\partial \omega} \leq 0, \frac{\partial \widehat{S}_{j I}^{\dagger}}{\partial \omega} \leq 0, \frac{\partial \widehat{W}_{i j}^{\dagger}}{\partial \omega} \leq 0 .
$$

In sharp contrast, a similar derivation for local equivalent properties that neglect the electromomentum coupling will result in a description that violates the physical requirements above. This is demonstrated in Fig. 6, where we show $\mathbf{A}_{\mathrm{eq}}$ as function of the normalized frequency. Specifically, in panel a we show $\mathbf{A}_{\text {eq }}^{\prime}$, and observe that the components $A_{\text {eq11 }}^{\prime}$ and $A_{\text {eq } 12}^{\prime}$ have a negative slope; and in panel b we show $\mathbf{A}_{\text {eq }}$, and observe it is nonzero with a negative slope ${ }^{9}$.

Interestingly, a similar demonstration of the need for the inclusion of the electromomentum coupling in order to obtain a physical model was shown by Pernas-Salomón et al. [51]. There, the authors used a heuristic homogenization approach, based on the scattering response of an asymmetric piezoelectric element.

\footnotetext{
${ }^{9}$ The requirement that in the local limit, the dielectric tensor of electromagnetic media satisfies $\partial \tilde{A}_{i j} / \partial \omega \geq 0$ can be found in Ref. [25]; the extension of this condition to generalized Willis media was derived in Ref. [50].
} 
(a)

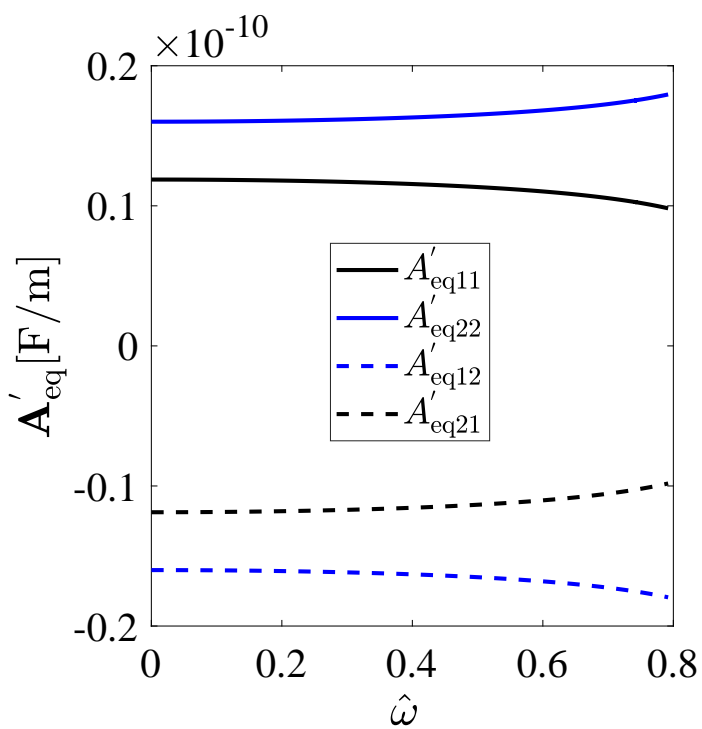

(b)

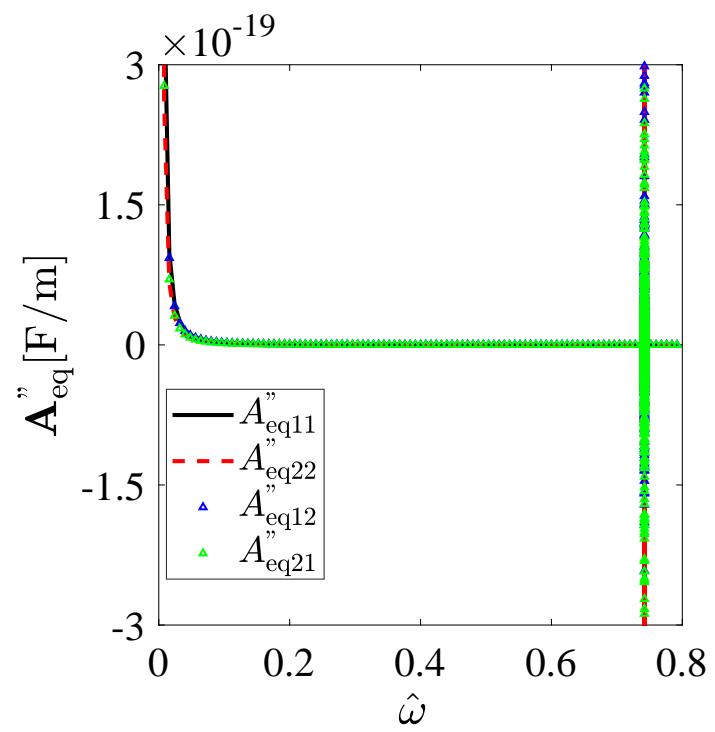

Fig. 6: The local approximation $(\boldsymbol{\kappa}=\mathbf{0})$ of the (a) real and (b) imaginary parts of the equivalent dielectric tensor as functions of the normalized frequency, calculated for PZT4 circular sector fibers in a PMMA matrix (Tab. 1).

\subsection{Effective properties outside the dispersion curve}

We recall that in the presence of sources, the frequency and the effective wavevector are independent one of the other. We demonstrate next the calculations of the effective properties in such case, i.e., outside the dispersion curves. By way of example, in Fig. 7 we examine the case $\kappa_{1}=\pi / a, \kappa_{2}=0$, which corresponds to a wavelength that is twice the period of the medium, and evaluate the properties as functions of the normalized frequency. We observe that the normalized resonance frequency has shifted to $\approx 0.48$. We observe that the effective properties satisfy Eq. (32) and except $\widehat{W}$ and $\widehat{S}$ are all reals as they should be.

\section{Summary}

Rigorous homogenization theories are key in understanding the physics of heterogeneous media, and hence in the development of metamaterials. These theories have shown how composites can exhibit nonlocal effective properties that do not exist in their constituents $[4,16,17,49,73,75]$. Here, we have developed explicit formulae to the homogenization theory of Pernas-Salomón and Shmuel [49], specialized for antiplane shear waves in fibrous piezoelectric composites.

The formulae above are more accessible than the previous ones, since here we rely on the plane wave expansion method in the averaging process, instead of the Green's function method in Ref. [49], the knowledge of which is often unknown. Both formulations follow two principles that were advocated in rigorous electromagnetic and elastodynamics homogenization theories 
(a)

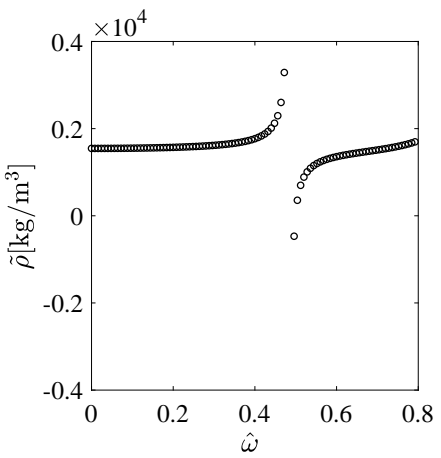

(d)

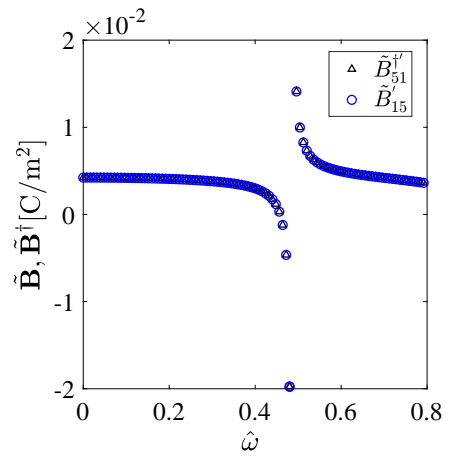

(g) (b)

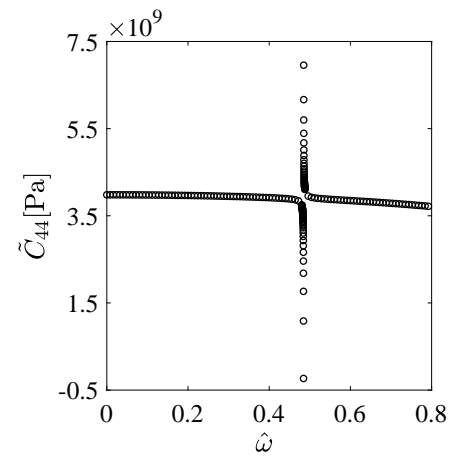

(e)

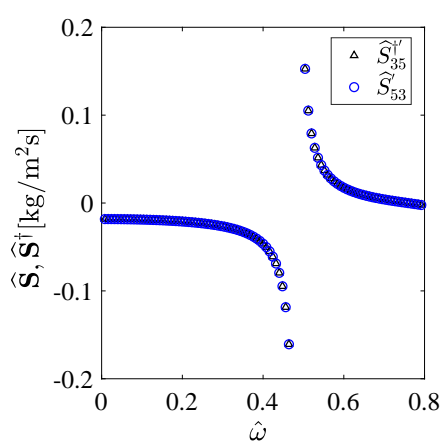

(c)

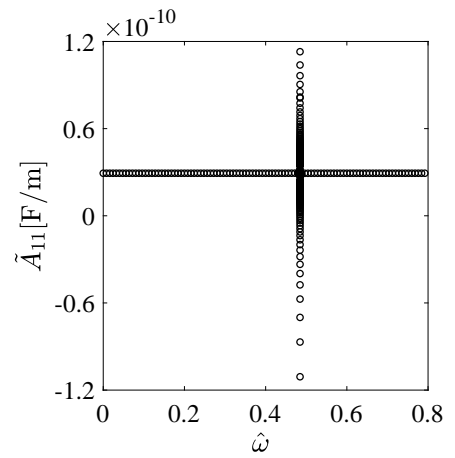

(f)

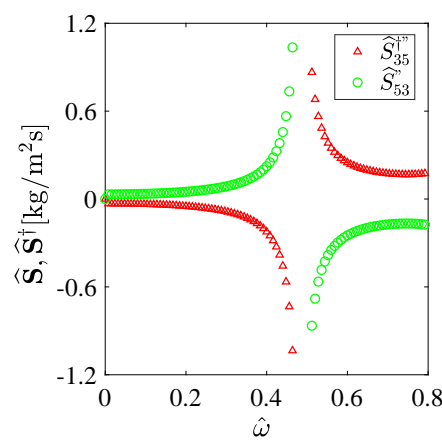

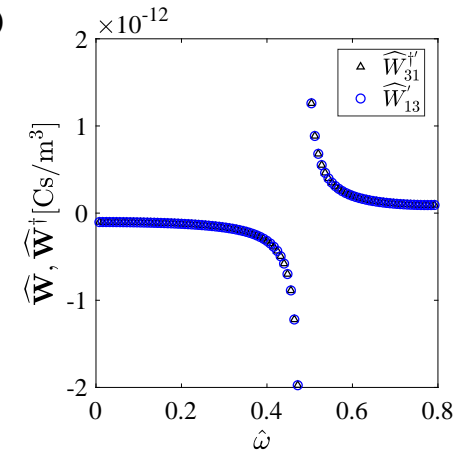

(h)

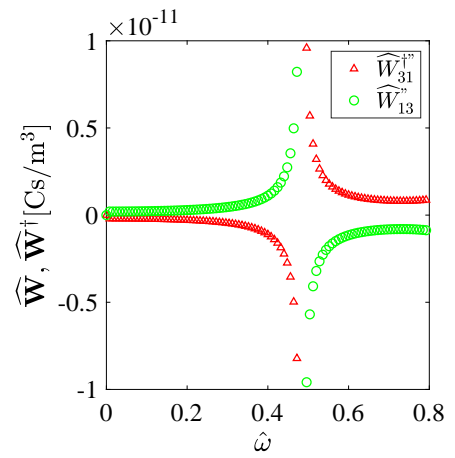

Fig. 7: Effective properties as functions of the normalized frequency outside the dispersion curves at the wavevector $\kappa_{1}=\pi / a, \kappa_{2}=0$, calculated for circular sector fibers in a PMMA matrix (Tab. 1). (a) Mass density; and selected components of the (b) elastic; (c) dielectric; and (d) piezoelectric tensors. Panels e and f (resp. $g$ and $h$ ) show the real and imaginary parts of the Willis tensor (resp. the electromomentum tensor). 
$[3,4,16,17,47,57,73,75]$. First, the volume averaging over the microscopic Bloch-Floquet waves is carried out only over their periodic part. Second, the microscopic governing equations and constitutive relations include driving sources. The reason for considering them is mainly mathematical: while these sources enter our formulation in a form that is challenging to access experimentally, if at all possible, they allow us to derive a unique set of effective properties that is applicable for general frequency-wavevector pairs, not only for those that are related by the normal modes of the composite. Importantly, the resultant effective properties are physically meaningful, since they satisfy the mathematical restrictions that reciprocity, causality and energy conservation impose [50].

We have evaluated our formulae for two composites made of PZT4 inclusions in a PMMA matrix. The tensorial nature of the problem has allowed us to demonstrate certain symmetries of the effective properties that cannot be observed in one-dimensional problems. Our numerical examples have shown that the effective medium recovers the exact dispersion of free waves in the original composites. We have also calculated the approximated local effective properties, and the nonlocal effective properties at representative arbitrary frequency-wavevector pairs. Our computations demonstrate how the effective properties indeed respect reciprocity, causality and energy conservation.

When sources are not considered, i.e., for free waves, it is possible to define alternative homogenized descriptions that also recover the dispersion relations of the original composite. In spite of the fact that such descriptions produce this particular measurable quantity properly, they are not admissible, since they generally predict nonreciprocal, nonconservative response for reciprocal, conservative media. Such violations were discussed in detail in Refs. [3, 4, 57], who studied analogous electromagnetic and acoustic problems. Lending the terminology in these works, we referred to the alternative models that neglect cross-couplings of Willis type as equivalent models. As explained in Refs. $[4,57,60]$, while the secondary properties (impedance and wavevector) of the effective and equivalent sets coincide, the primary properties of the equivalent set lack physical meaning. We have evaluated the properties of the equivalent model for our case study, and showed that indeed they (more specifically, the equivalent dielectric tensor) violate reciprocity, causality and energy conservation.

It is imperative to examine the implications of these homogenization results—derived for an infinite medium - in predicting the scattering response of a finite piezoelectric medium with asymmetric microstructure (cf. the analysis and applications in Refs. [22, 27, 48, 53, 63] for acoustic and elastic problems). In one-dimensional scattering problems, Pernas-Salomón et al. [51] showed that the resultant electromomentum coupling is related to directional phase angle that depends on the electric circuit conditions; such a study in two-dimensional settings as considered here is left for future work. 


\section{Acknowledgments}

This research was supported by the Israel Science Foundation, Israel Academy of Sciences and Humanities (Grant no. 2061/20), the United States-Israel Binational Science Foundation (Grant no. 2014358), and Ministry of Science and Technology (grant no. 880011). D.T. acknowledges financial support through the "Ramón y Cajal" fellowship under Grant No. RYC-2016-21188 and from the Ministry of Science, Innovation and Universities through Project No. RTI2018- 093921A-C42.

\section{Appendix A. Explicit expressions for the matrices in Sec. 3.}

The description of matrices is given here

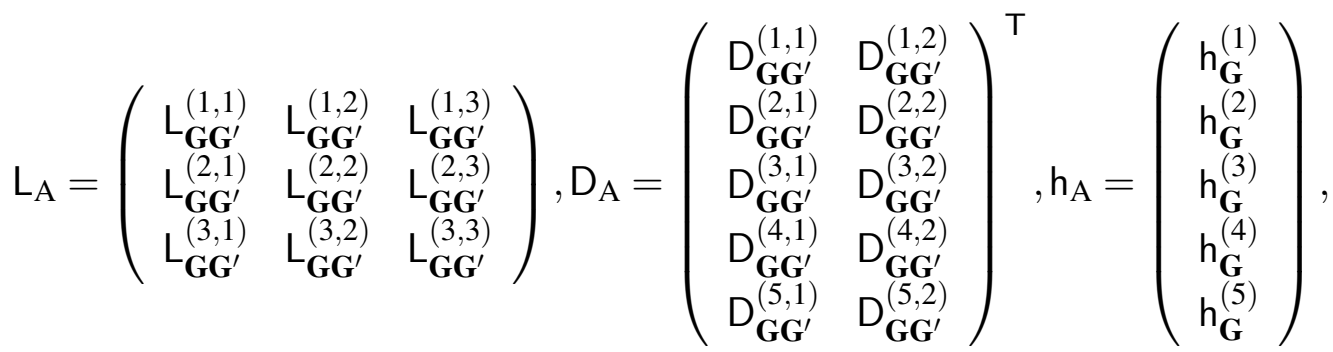

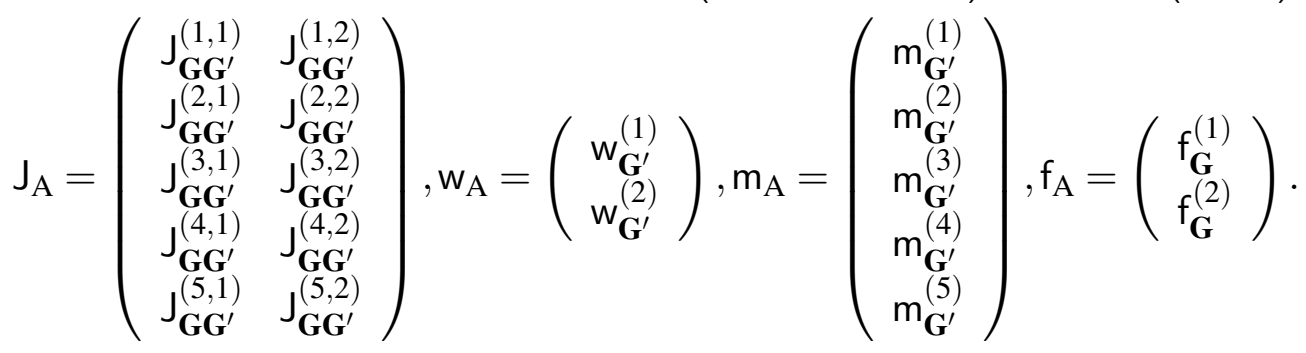

where the components of the assembly matrix $L_{A}$ are

$$
\begin{aligned}
& \mathrm{L}_{\mathbf{G} \mathbf{G}^{\prime}}^{(1,1)}=\check{\mathrm{C}}_{\mathbf{G G}^{\prime}}, \mathrm{L}_{\mathbf{G G}^{\prime}}^{(1,2)}=\check{\mathrm{B}}_{\mathbf{G G}^{\prime}}^{\top}, \\
& \mathrm{L}_{\mathbf{G G}^{\prime}}^{(2,1)}=\check{\mathrm{B}}_{\mathbf{G G}^{\prime}}, \mathrm{L}_{\mathbf{G G}^{\prime}}^{(2,2)}=-\check{\mathrm{A}}_{\mathbf{G}} \mathbf{G}^{\prime}, \\
& \mathrm{L}_{\mathbf{G G}^{\prime}}^{(3,3)}=\check{\rho}_{\mathbf{G G}^{\prime}}, \mathrm{L}_{\mathbf{G G}^{\prime}}^{(1,3)}=\mathrm{L}_{\mathbf{G G}^{\prime}}^{(2,3)}=\mathrm{L}_{\mathbf{G G}^{\prime}}^{(3,1)}=\mathrm{L}_{\mathbf{G G}^{\prime}}^{(3,2)}=0, \\
& \mathrm{D}_{\mathbf{G G}^{\prime}}^{(1,1)}=\mathrm{D}_{\mathbf{G G}^{\prime}}^{(3,2)}=i\left(\kappa_{1}+G_{1}\right) \check{\delta}_{\mathbf{G G}^{\prime}}, \\
& \mathrm{D}_{\mathbf{G G}^{\prime}}^{(2,1)}=\mathrm{D}_{\mathbf{G G}^{\prime}}^{(4,2)}=i\left(\kappa_{2}+G_{2}\right) \check{\delta}_{\mathbf{G G}^{\prime}}, \\
& \mathrm{D}_{\mathbf{G G}^{\prime}}^{(5,1)}=i \omega \check{\delta}_{\mathbf{G G}^{\prime}}, \\
& \mathrm{D}_{\mathbf{G G}^{\prime}}^{(1,2)}=\mathrm{D}_{\mathbf{G G}^{\prime}}^{(2,2)}=\mathrm{D}_{\mathbf{G G}^{\prime}}^{(3,1)}=\mathrm{D}_{\mathbf{G G}^{\prime}}^{(4,1)}=\mathrm{D}_{\mathbf{G G}^{\prime}}^{(5,2)}=0,
\end{aligned}
$$




$$
\begin{gathered}
J_{\mathbf{G}^{\prime}}^{(1,1)}=J_{\mathbf{G G}^{\prime}}^{(3,2)}=i\left(\kappa_{1}+G_{1}\right) \check{\delta}_{\mathbf{G G}^{\prime}}, \\
\mathrm{J}_{\mathbf{G}^{\prime}}^{(2,1)}=J_{\mathbf{G G}^{\prime}}^{(4,2)}=i\left(\kappa_{2}+G_{2}\right) \check{\delta}_{\mathbf{G G}^{\prime}}, \\
\mathrm{J}_{\mathbf{G}^{\prime}}^{(5,1)}=-i \omega \check{\delta}_{\mathbf{G}^{\prime}}, \\
J_{\mathbf{G}^{\prime}}^{(1,2)}=J_{\mathbf{G}^{\prime}}^{(2,2)}=J_{\mathbf{G}^{\prime}}^{(3,1)}=J_{\mathbf{G}^{\prime}}^{(4,1)}=J_{\mathbf{G G}^{\prime}}^{(5,2)}=0, \\
\mathrm{~h}_{\mathbf{G}}^{(1)}=\check{\sigma}_{\mathbf{G}}^{13}, \mathrm{~h}_{\mathbf{G}}^{(2)}=\check{\sigma}_{\mathbf{G}}^{23}, \mathrm{~h}_{\mathbf{G}}^{(3)}=\check{D}_{\mathbf{G}}^{1}, \mathrm{~h}_{\mathbf{G}}^{(4)}=\check{D}_{\mathbf{G}}^{2}, \mathrm{~h}_{\mathbf{G}}^{(5)}=\check{p}_{\mathbf{G}}, \\
\mathrm{w}_{\mathbf{G}^{\prime}}^{(1)}=\check{u}_{\mathbf{G}^{\prime}}, \mathrm{w}_{\mathbf{G}^{\prime}}^{(2)}=\check{\phi}_{\mathbf{G}^{\prime}}, \\
\mathrm{f}_{\mathbf{G}}^{(1)}=\check{f}_{\mathbf{G}}, \mathrm{f}_{\mathbf{G}}^{(2)}=-\check{q}_{\mathbf{G}}, \\
\mathrm{m}_{\mathbf{G}^{\prime}}^{(1)}=\check{\eta}_{\mathbf{G}^{\prime}}^{1}, \mathrm{~m}_{\mathbf{G}^{\prime}}^{(2)}=\check{\eta}_{\mathbf{G}^{\prime}}^{2}, \mathrm{~m}_{\mathbf{G}^{\prime}}^{(3)}=0, \mathrm{~m}_{\mathbf{G}^{\prime}}^{(4)}=\check{0}_{\mathbf{G}^{\prime}}, \mathrm{m}_{\mathbf{G}^{\prime}}^{(5)}=0 .
\end{gathered}
$$

\section{Appendix B. Validation of the homogenization scheme.}

In addition to the validating features listed in Sec. 4, the validity of our effective model is further supported by its predictions in the low frequency, long-wavelength limit. In this limit, the effective mass density of our model is equal to the volume average, as it should [31]. Furthermore, in this limit, there are also several benchmark results in the one-dimensional setting. To test our model against these results, we used our two-dimensional homogenization scheme to analyze a layered unit cell whose properties are uniform along $x_{2}$ and periodic along $x_{1}$. For this microstructure, we considered a wavevector whose $x_{2}$-component is null and its $x_{1}$-component is infinitely small, but greater than 0 . By doing so, we reduce our problem to a one-dimensional problem in the longwavelength limit of propagation in the periodicity direction. When taking $B(\mathbf{x}) \rightarrow 0$, we find that $\tilde{C}_{55}\left(:=\tilde{C}_{1313}\right)$ and $\tilde{A}_{11}$ are equal to their weighted harmonic mean, agreeing with their expected values from static homogenization in the limiting decoupled case. When taking nonzero $B(\mathbf{x})$, we find numerically that

$$
\tilde{C}_{55}=\left[\left\langle C_{\mathrm{D}}^{-1}\right\rangle+\gamma\left\langle\frac{B}{A C_{\mathrm{D}}}\right\rangle\right]^{-1}, \text { where } C_{\mathrm{D}}=C+B^{2} / A, \gamma=\left\langle\frac{B}{A C_{\mathrm{D}}}\right\rangle\left\langle\frac{C}{A C_{\mathrm{D}}}\right\rangle^{-1}
$$

Result (B.1) was obtained analytically by Pernas-Salomón et al. [51] using heuristic homogenization of one-dimensional piezoelectric scatterers. Pernas-Salomón et al. [51] also obtained that $(i)$ the local Willis modulus depends on the sum of the asymmetry in the elastic impedance $Z_{\mathrm{E}}^{2}=\rho C$, as well-known in the purely elastic case [42,57], and the asymmetry in a piezoelectric-like impedance, $\rho B$; (ii) the local electromomentum modulus depends on the asymmetry in the ratio $B / A$. Using a one-dimensional version of the scheme developed here, we have computed the effective prop- 
erties of a trilayer whose constituents are such $\rho B, \rho C$ and $B / A$ exhibit inversion symmetry; we received zero $\tilde{S}_{53}\left(:=\tilde{S}_{133}\right)$ and zero $\tilde{W}_{13}$. When breaking the symmetry of $B / A$ in the $x_{1}$-direction, we received nonzero $\tilde{W}_{13}$. Subsequently, we examined a case were the inversion symmetry of $\rho B$ is broken; indeed, this case resulted with nonzero $\tilde{S}_{53}$. The agreement in the above benchmarks between the two homogenization approaches reinforces their validity. We numerically observed, however, that in our periodic homogenization, if the mass density mass is spatially contrast, then both the Willis coupling and the electromomentum coupling are null; in the heuristic homogenization, this condition is not necessary.

Finally, we analyzed a second case, by considering a wavevector whose $x_{1}$-component is null and its $x_{2}$-component is infinitely small, but greater than 0 . By doing so, we reduce our problem to a one-dimensional problem in the long-wavelength limit of propagation along the layers. When taking $B(\mathbf{x}) \rightarrow 0$, we find that $\tilde{C}_{44}\left(:=\tilde{C}_{2323}\right)$ and $\tilde{A}_{22}$ are equal to their volume average, agreeing with their expected values from static homogenization in the limiting decoupled case.

\section{Appendix C. On nonlocal components in constitutive equations.}

As mentioned in the main text, nonlocality induces components in the effective tensor that do not appear in local constitutive equations, even if both media are isotropic. (Such components are somewhat overlooked in the literature, but they do exist.) For example, the constitutive relation of a statistically homogeneous nonlocal linear isotropic elastic solid is [1]

$$
\sigma_{i j}(\mathbf{x})=\int_{\Omega} C_{i j k l}\left(\mathbf{x}-\mathbf{x}^{\prime}\right) \varepsilon_{k l}\left(\mathbf{x}^{\prime}\right) \mathrm{d} \Omega,
$$

where $\boldsymbol{\varepsilon}$ is the strain and

$$
C_{i j k l}=\lambda \delta_{i j} \delta_{k l}+\mu\left(\delta_{i k} \delta_{j l}+\delta_{i l} \delta_{j k}\right)+\alpha\left(\kappa_{i} \kappa_{j} \delta_{k l}+\kappa_{k} \kappa_{l} \delta_{i j}\right)+\beta \kappa_{i} \kappa_{j} \kappa_{k} \kappa_{l},
$$

for some material moduli $\lambda, \mu, \alpha$ and $\beta$ that are functions of the translation $\left|\mathbf{x}-\mathbf{x}^{\prime}\right|$. The moduli $\alpha$ and $\beta$ multiply terms that vanish in local isotropic solids. Thus, by way of example, the component $C_{1234}$ must be null in local isotropic solids, while having a finite value for $\boldsymbol{\kappa}>\mathbf{0}$. Analogous results hold for electromagnetic constitutive relations, see, e.g., Eq. (3.1.21) in Ref. [2]. 


\section{Appendix D. The dispersion relation of the homogenized medium.}

Within an effective elastic-piezoelectric medium, the governing equations are

$$
\begin{aligned}
& \nabla \cdot\langle\boldsymbol{\sigma}\rangle=-i \omega\langle\mathbf{p}\rangle, \\
& \nabla \cdot\langle\mathbf{D}\rangle=0 .
\end{aligned}
$$

We can rewrite Eq. (D.1) for our problem as

$$
\begin{aligned}
& i \kappa^{\top}\left(\begin{array}{l}
\left\langle\sigma^{13}\right\rangle \\
\left\langle\sigma^{23}\right\rangle
\end{array}\right)=-i \omega\langle p\rangle, \\
& i \kappa^{\top}\left(\begin{array}{l}
\left\langle D^{1}\right\rangle \\
\left\langle D^{2}\right\rangle
\end{array}\right)=0 .
\end{aligned}
$$

where $\mathrm{K}$ is a column vector with 2 components. The constitutive relations are

$$
\left\{\begin{array}{c}
\langle\sigma\rangle=\tilde{\mathrm{C}} i \kappa\langle u\rangle+\tilde{\mathrm{B}}^{\dagger} i \kappa\langle\phi\rangle-\tilde{\mathrm{S}}\langle i \omega u\rangle, \\
\langle\mathrm{d}\rangle=\tilde{\mathrm{B}} i \kappa\langle u\rangle-\tilde{\mathrm{A}} i \kappa\langle\phi\rangle-\tilde{\mathrm{W}}\langle i \omega u\rangle, \\
\langle p\rangle=\tilde{\mathrm{S}}^{\dagger} i \kappa\langle u\rangle+\tilde{\mathrm{W}}^{\dagger} i \kappa\langle\phi\rangle-\tilde{\rho}\langle i \omega u\rangle,
\end{array}\right.
$$

where $\mathrm{d}$ is the represented vector which contains the electric displacement terms $D^{1}$ and $D^{2}$. Using Eq. (D.2) and the constitutive relations we can write the relation between the displacement and the electric potential, namely,

$$
\kappa^{\top} \tilde{\mathrm{B}} \kappa\langle u\rangle-\kappa^{\top} \tilde{\mathrm{A}} \kappa\langle\phi\rangle-\kappa^{\top} \tilde{\mathrm{W}} \omega\langle u\rangle=0,
$$

we rearrange the equation

$$
\kappa^{\top} \tilde{\mathrm{B}} \kappa\langle u\rangle-\kappa^{\top} \tilde{\mathrm{W}} \omega\langle u\rangle=\kappa^{\top} \tilde{\mathrm{A}} \kappa\langle\phi\rangle .
$$

We notice that $\kappa^{\top} \tilde{A} \kappa$ is a scalar to obtain

$$
\frac{\kappa^{\top} \tilde{\mathrm{B}} \kappa-\kappa^{\top} \tilde{\mathrm{W}} \omega}{\kappa^{\top} \tilde{\mathrm{A}} \kappa}\langle u\rangle=\langle\phi\rangle .
$$

Our goal is to find the equation that we will use to recover the dispersion relation for any prescribed к. To this end, We use Eq. (D.2) to obtain

$$
i \kappa^{\top}\left(\tilde{\mathrm{C}} i \kappa\langle u\rangle+\tilde{\mathrm{B}}^{\dagger} i \kappa\langle\phi\rangle-\tilde{\mathrm{S}}\langle i \omega u\rangle\right)=-i \omega\left(\tilde{\mathrm{S}}^{\dagger} i \kappa\langle u\rangle+\tilde{\mathrm{W}}^{\dagger} i \kappa\langle\phi\rangle-\tilde{\rho}\langle i \omega u\rangle\right)
$$


We rearrange the equation to get quadratic equation that will allow us to get the dispersion relation

$$
a \omega^{2}+b \omega+c=0
$$

where $a, b$ and $c$ are

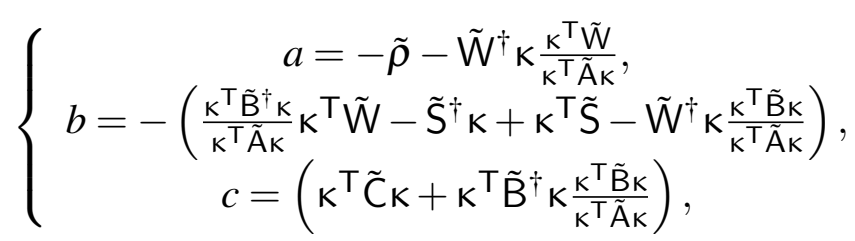

such that

$-\left(\tilde{\rho}+\tilde{W}^{\dagger} \kappa \frac{\kappa^{\top} \tilde{W}}{\kappa^{\top} \tilde{A} \kappa}\right) \omega^{2}-\left(\frac{\kappa^{\top} \tilde{B}^{\dagger} \kappa}{\kappa^{\top} \tilde{A} \kappa} \kappa^{\top} \tilde{W}-\tilde{S}^{\dagger} \kappa+\kappa^{\top} \tilde{S}-\tilde{W}^{\dagger} \kappa \frac{\kappa^{\top} \tilde{B} \kappa}{\kappa^{\top} \tilde{A} \kappa}\right) \omega+\left(\kappa^{\top} \tilde{C} \kappa+\kappa^{\top} \tilde{B}^{\dagger} \kappa \frac{\kappa^{\top} \tilde{B} \kappa}{\kappa^{\top} \tilde{A} \kappa}\right)=0$.

Using Eq. (D.10) we can obtain the eigenfrequencies that corresponds to the effective properties. The first two branches that are fully recovered are shown in Fig. 3. The dispersion relation of the composite was calculate from the eigenvalue problem $\operatorname{det}\left(D_{A}^{\top} L_{A} J_{A}\right)=0$.

\section{Appendix E. Additional components of the effective properties for the asymmetric cell.}

Fig. 8 presents additional effective properties for the asymmetric cell in the local approximation. Fig. 9 presents additional effective properties for the asymmetric cell when the 1-component of wavevector is $\kappa_{1}=\pi / a$ and 2-component is null.

\section{References}

[1] Nonlocal Continuum Field Theories. Springer, New York. Springer, New York. Springer, 2002.

[2] V. M. Agranovich and V. L. Ginzburg. Crystal Optics with Spatial Dispersion, and Excitons, volume 42. Springer Series in Solid-State Sciences, Berlin, 1984.

[3] Andrea Alù. Restoring the physical meaning of metamaterial constitutive parameters. Phys. Rev. B, 83:081102, Feb 2011. doi: 10.1103/PhysRevB.83.081102. URL https: / / I ink . aps.org/doi/10.1103/PhysRevB.83.081102. 
(a)

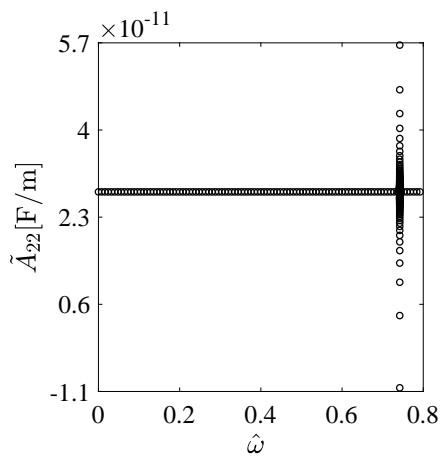

(d)

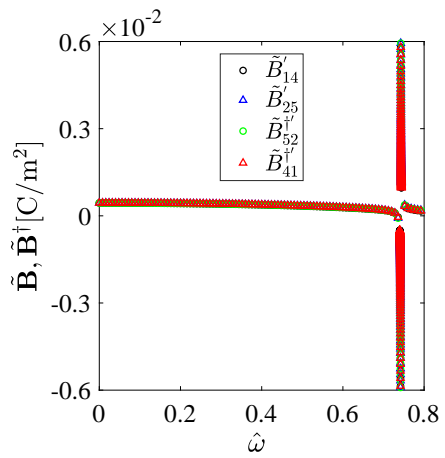

(b)

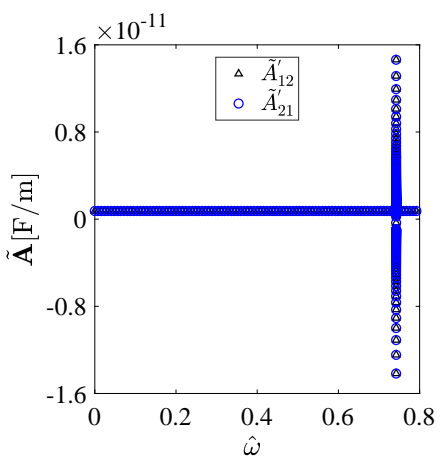

(e)

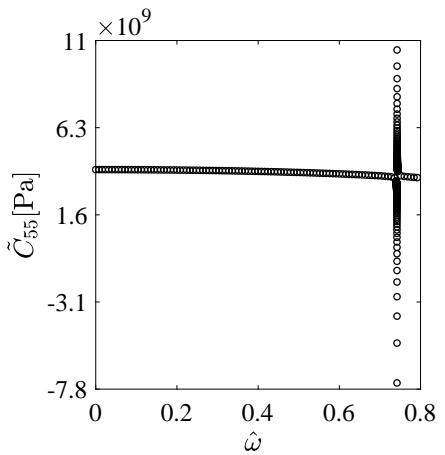

(c)

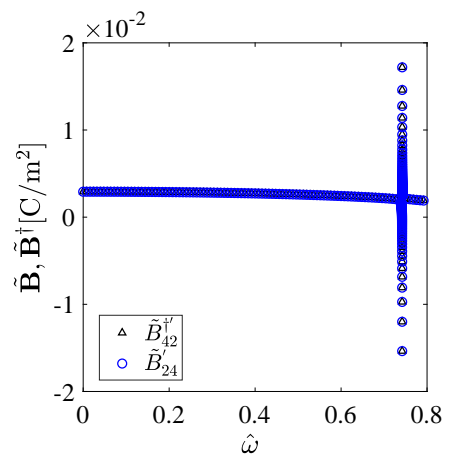

(f)

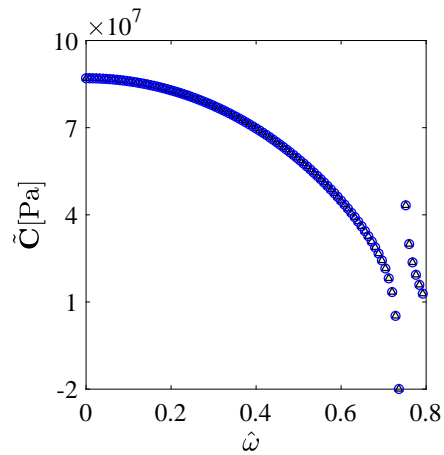

(g)

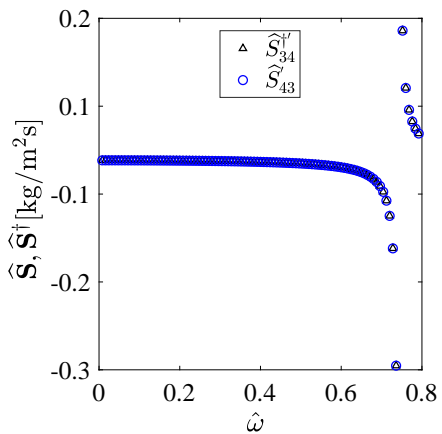

(h)

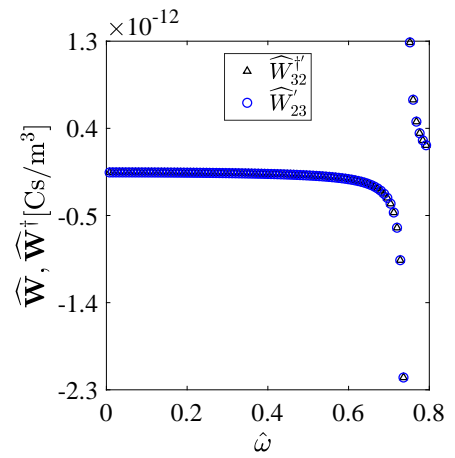

Fig. 8: Selected components of the (a,b) dielectric; (c,d) piezoelectric; (e,f) elastic; (g) Willis; and (h) electromomentum tensors of PZT4 circular sector fibers in a PMMA matrix Tab. 1 for the local approximation $(\boldsymbol{\kappa}=\mathbf{0})$ case, as function of the normalized frequency. 
(a)

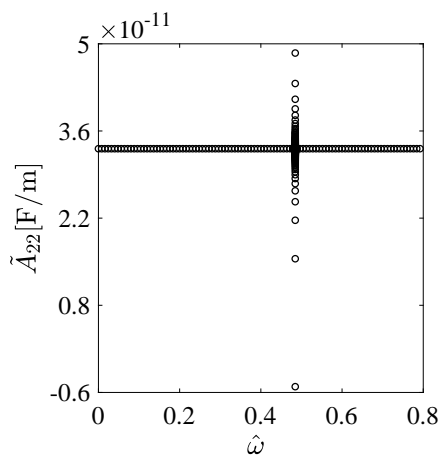

(d)

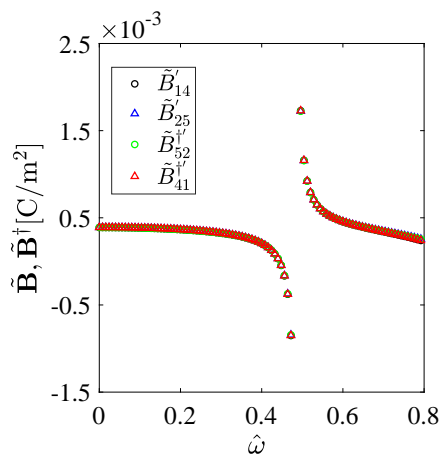

(g)

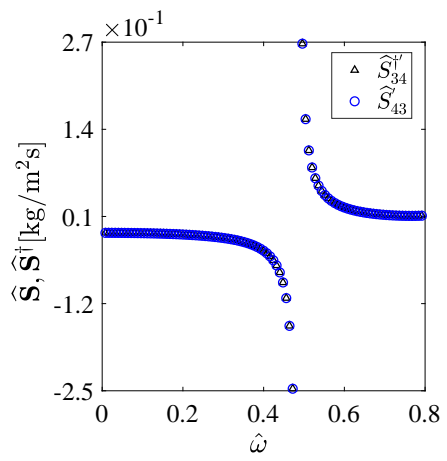

(b)

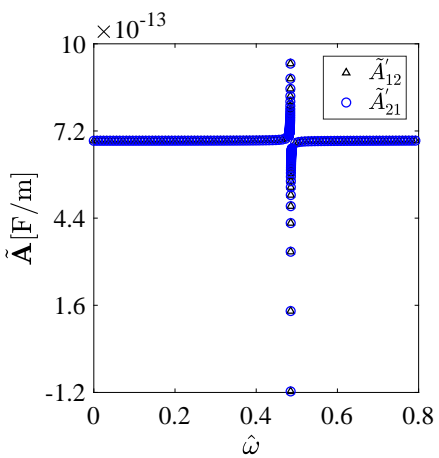

(e)

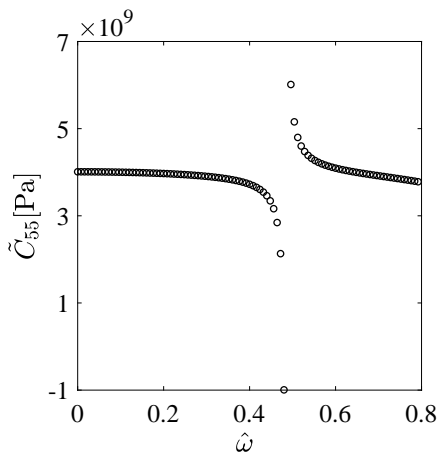

(h)

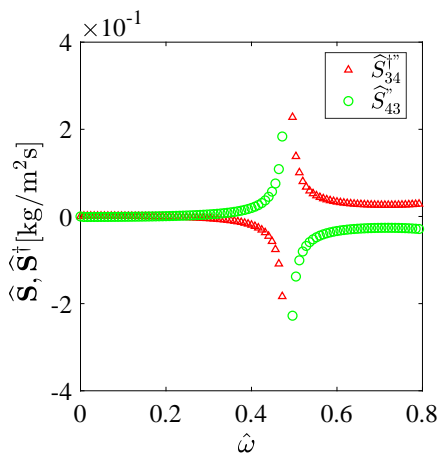

(j)

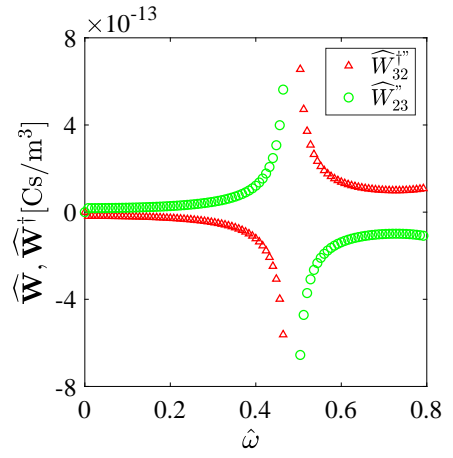

(c)

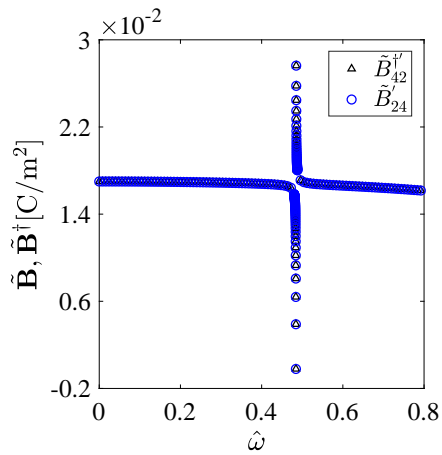

(f)

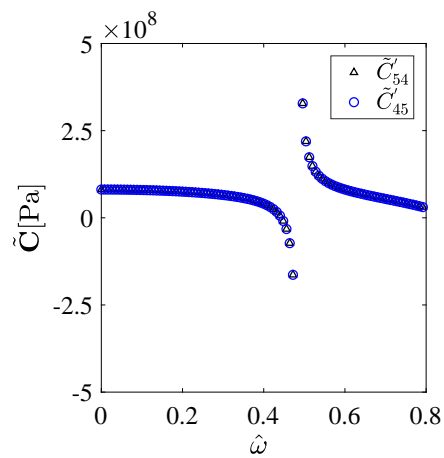

(i)

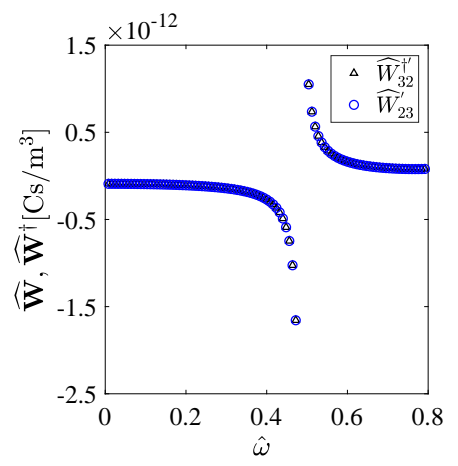

Fig. 9: Selected components of the (a,b) dielectric; (c,d) piezoelectric; (e,f) elastic; (g,h) Willis; and $(\mathrm{i}, \mathrm{j})$ electromomentum tensors of PZT4 circular sector fibers in a PMMA matrix Tab. 1 for the outside of the dispersion case $\left(\kappa_{1}=\pi / a, \kappa_{2}=0\right)$, as function of the normalized frequency. 
[4] Andrea Alù. First-principles homogenization theory for periodic metamaterials. Phys. Rev. B, 84:075153, Aug 2011. doi: 10.1103/PhysRevB.84.075153. URL https:// link. aps. org/doi/10.1103/PhysRevB.84.075153.

[5] T Antonakakis, R V Craster, and S Guenneau. Asymptotics for metamaterials and photonic crystals. Proc. R. Soc. London A Math. Phys. Eng. Sci., 469(2152), 2013. ISSN 1364-5021. doi: 10.1098/rspa.2012.0533. URL http://rspa.royalsocietypublishing. org/content/469/2152/20120533.

[6] E G Barnwell, W J Parnell, and I D Abrahams. Antiplane elastic wave propagation in pre-stressed periodic structures; tuning, band gap switching and invariance. Wave Motion, 63:98-110, 2016. ISSN 0165-2125. doi: http://dx.doi.org/10.1016/j.wavemoti. 2016.02.001. URL http://www.sciencedirect.com/science/article/pii/ S0165212516000111.

[7] S. Bolioli. Bi-Isotropic and Bi-Anisotropic Media, pages 33-51. Springer Netherlands, Dordrecht, 1997. ISBN 978-94-011-5734-6. doi: 10.1007/978-94-011-5734-6\{\\}3. URL https://doi.org/10.1007/978-94-011-5734-6_3.

[8] Mihai Caleap and Bruce W Drinkwater. Metamaterials: supra -classical dynamic homogenization. New J. Phys., 17(12):123022, 2015. URL http://stacks.iop.org/ $1367-2630 / 17 / i=12 / a=123022$.

[9] Yangyang Chen, Xiaopeng Li, Gengkai Hu, Michael R. Haberman, and Guoliang Huang. An active mechanical Willis meta-layer with asymmetric polarizabilities. Nature Communications, 11(1):3681, 2020. doi: 10.1038/s41467-020-17529-2. URL https://doi.org/ $10.1038 / \mathrm{s} 41467-020-17529-2$.

[10] Choonlae Cho, Xinhua Wen, Namkyoo Park, and Jensen Li. Digitally virtualized atoms for acoustic metamaterials. Nature Communications, 11(1):251, 2020. doi: 10.1038/ s41467-019-14124-y. URL https://doi.org/10.1038/s41467-019-14124-y.

[11] Choonlae Cho, Xinhua Wen, Namkyoo Park, and Jensen Li. Acoustic willis metaatom beyond the bounds of passivity and reciprocity. Communications Physics, 4(1): 82, 2021. doi: 10.1038/s42005-021-00584-6. URL https://doi.org/10.1038/ s42005-021-00584-6.

[12] Johan Christensen, Muamer Kadic, Oliver Kraft, and Martin Wegener. Vibrant times for mechanical metamaterials. MRS Communications, 5:453-462, 2015. doi: 10.1557/mrc.2015. 51. 
[13] D Cioranescu and P Donato. An introduction to homogenization. Oxford University Press, Oxford, UK, 1999.

[14] Richard V Craster and Sébastien Guenneau. Acoustic metamaterials: Negative refraction, imaging, lensing and cloaking, volume 166. Springer Science \& Business Media, 2012.

[15] Pierre A Deymier. Acoustic metamaterials and phononic crystals, volume 173. Springer Science \& Business Media, 2013.

[16] C. Fietz and G. Shvets. Metamaterial homogenization: extraction of effective constitutive parameters. Proc. SPIE 7392, Metamaterials: Fundamentals and Applications II, page 73920L, 2009. doi: 10.1117/12.827031.

[17] Chris Fietz and Gennady Shvets. Current-driven metamaterial homogenization. Physica B: Condensed Matter, 405(14):2930 - 2934, 2010. ISSN 0921-4526. doi: https://doi.org/ 10.1016/j.physb.2010.01.006. URL http://www.sciencedirect.com/science/ article/pii/s0921452610000086. Proceedings of the Eighth International Conference on Electrical Transport and Optical Properties of Inhomogeneous Media.

[18] R Getz and G Shmuel. Band gap tunability in deformable dielectric composite plates. Int. J. Solids Struct., 2017. ISSN 0020-7683. doi: https://doi.org/10.1016/j.ijsolstr. 2017.07.021. URL http: / / www. sciencedirect.com/science/article/pii/ s 0020768317303384.

[19] R Getz, D M Kochmann, and G Shmuel. Voltage-controlled complete stopbands in twodimensional soft dielectrics. Int. J. Solids Struct., 113-114:24-36, 2017. ISSN 0020-7683. doi: http://doi.org/10.1016/j.ijsolstr.2016.10.002. URL http: / / www . sciencedirect . com/science/article/pii/s0020768316302931.

[20] Z Hashin. Analysis of composite materials - A survey. J. Appl. Mech., 50:481-505, 1983.

[21] Muamer Kadic, Graeme W. Milton, Martin van Hecke, and Martin Wegener. 3d metamaterials. Nature Reviews Physics, 1(3):198-210, 2019. doi: 10.1038/s42254-018-0018-y. URL https://doi.org/10.1038/s42254-018-0018-y.

[22] Sukmo Koo, Choonlae Cho, Jun-ho Jeong, and Namkyoo Park. Acoustic omni meta-atom for decoupled access to all octants of a wave parameter space. Nature Communications, 7:13012 EP -, 09 2016. URL https: / / doi .org/10 . 1038 / ncomms 13012.

[23] C. É. Kriegler, M. S. Rill, S. Linden, and M. Wegener. Bianisotropic photonic metamaterials. IEEE Journal of Selected Topics in Quantum Electronics, 16(2):367-375, March 2010. ISSN 1077-260X. doi: 10.1109/JSTQE.2009.2020809. 
[24] M S Kushwaha, P Halevi, L Dobrzynski, and B Djafari-Rouhani. Acoustic band structure of periodic elastic composites. Phys. Rev. Lett., 71(13):2022-2025, 1993.

[25] Lev Davidovich Landau, Evgenii Mikhailovich Lifshitz, and Lev Petrovich Pitaevskii. Course of theoretical physics; 2nd ed. Butterworth, Oxford, 1984. URL https://cds.cern. $\mathrm{ch} / \mathrm{record} / 712712$.

[26] Joshua Lau, Suet To Tang, Min Yang, and Zhiyu Yang. Coupled Decorated Membrane Resonators with Large Willis Coupling. Phys. Rev. Applied, 12:014032, Jul 2019. doi: 10.1103/PhysRevApplied.12.014032. URL https://link.aps.org/doi/10 . 1103/PhysRevApplied.12.014032.

[27] Junfei Li, Chen Shen, Ana D'iaz-Rubio, Sergei A Tretyakov, and Steven A Cummer. Systematic design and experimental demonstration of bianisotropic metasurfaces for scatteringfree manipulation of acoustic wavefronts. Nat. Commun., 9(1):1342, 2018. doi: 10.1038/ s41467-018-03778-9. URL https://doi.org/10.1038/s41467-018-03778-9.

[28] Chenchen Liu and Celia Reina. Variational coarse-graining procedure for dynamic homogenization. Journal of the Mechanics and Physics of Solids, 104:187-206, 2017.

[29] Yongquan Liu, Zixian Liang, Jian Zhu, Lingbo Xia, Olivier Mondain-Monval, Thomas Brunet, Andrea Alù, and Jensen Li. Willis metamaterial on a structured beam. Phys. Rev. X, 9:011040, Feb 2019. doi: 10.1103/PhysRevX.9.011040. URL https:// link.aps . org/doi/10.1103/PhysRevX.9.011040.

[30] G Ma and P Sheng. Acoustic metamaterials: From local resonances to broad horizons. Sci. Adv., 2(2), 2016. doi: 10.1126/sciadv.1501595. URL http://advances. sciencemag.org/content/2/2/e1501595.

[31] P. A. Martin, A. Maurel, and W. J. Parnell. Estimating the dynamic effective mass density of random composites. The Journal of the Acoustical Society of America, 128(2):571577, 2021/07/12 2010. doi: 10.1121/1.3458849. URL https://doi.org/10.1121/ 1.3458849 .

[32] Anton Melnikov, Yan Kei Chiang, Li Quan, Sebastian Oberst, Andrea Alù, Steffen Marburg, and David Powell. Acoustic meta-atom with experimentally verified maximum Willis coupling. Nature Communications, 10(1):3148, 2019. doi: 10.1038/s41467-019-10915-5. URL https://doi.org/10.1038/s41467-019-10915-5.

[33] Shixu Meng and Bojan B Guzina. On the dynamic homogenization of periodic media: Willis' approach versus two-scale paradigm. Proc. R. Soc. London A Math. Phys. Eng. 
Sci., 474(2213), 2018. ISSN 1364-5021. doi: 10.1098/rspa.2017.0638. URL http: //rspa.royalsocietypublishing.org/content/474/2213/20170638.

[34] Aurélien Merkel, Vicent Romero-García, Jean-Philippe Groby, Jensen Li, and Johan Christensen. Unidirectional zero sonic reflection in passive $\mathscr{P} \mathscr{T}$-symmetric Willis media. Phys. Rev. B, 98:201102, Nov 2018. doi: 10.1103/PhysRevB.98.201102. URL https : / I ink . aps.org/doi/10.1103/PhysRevB.98.201102.

[35] G W Milton and J R Willis. On modifications of Newton's second law and linear continuum elastodynamics. Proc. R. Soc. London A Math. Phys. Eng. Sci., 463(2079):855880, 2007. ISSN 1364-5021. doi: 10.1098/rspa.2006.1795. URL http://rspa. royalsocietypublishing.org/content/463/2079/855.

[36] G W Milton, M Briane, and J R Willis. On cloaking for elasticity and physical equations with a transformation invariant form. New J. Phys., 8(10):248, 2006. URL http: / / stacks . iop.org $/ 1367-2630 / 8 / i=10 / a=248$.

[37] Graeme W Milton. The theory of composites, volume 6. Cambridge university press, 2002.

[38] Graeme W Milton. New metamaterials with macroscopic behavior outside that of continuum elastodynamics. New Journal of Physics, 9(10):359-359, oct 2007. doi: 10.1088/1367-2630/ 9/10/359. URL https://doi.org/10.1088\%2F1367-2630\%2F 9\%2F10\%2F359.

[39] Graeme W. Milton. A unifying perspective on linear continuum equations prevalent in physics. part ii: Canonical forms for time-harmonic equations. arXiv: Analysis of PDEs, 2020.

[40] Graeme W. Milton. A unifying perspective on linear continuum equations prevalent in physics. part iv: Canonical forms for equations involving higher order gradients. arXiv: Mathematical Physics, 2020.

[41] Michael B Muhlestein, Caleb F Sieck, Andrea Alù, and Michael R Haberman. Reciprocity, passivity and causality in Willis materials. Proc. R. Soc. London A Math. Phys. Eng. Sci., 472(2194), 2016. ISSN 1364-5021. doi: 10.1098/rspa.2016.0604. URL http://rspa. royalsocietypublishing.org/content/472/2194/20160604.

[42] Michael B. Muhlestein, Caleb F. Sieck, Preston S. Wilson, and Michael R. Haberman. Experimental evidence of Willis coupling in a one-dimensional effective material element. $\mathrm{Na}$ ture Communications, 8:15625 EP -, 06 2017. URL https://doi.org/10.1038/ ncomms 15625. 
[43] Michael B. Muhlestein, Benjamin M. Goldsberry, Andrew N. Norris, and Michael R. Haberman. Acoustic scattering from a fluid cylinder with Willis constitutive properties. Proceedings of the Royal Society A: Mathematical, Physical and Engineering Sciences, 474(2220):20180571, 2018. doi: 10.1098/rspa.2018.0571. URL https:// royalsocietypublishing.org/doi/abs/10.1098/rspa.2018.0571.

[44] H Nassar, X C Xu, A N Norris, and G L Huang. Modulated phononic crystals: Nonreciprocal wave propagation and Willis materials. Journal of the Mechanics and Physics of Solids, 101:10-29, 2017. ISSN 0022-5096. doi: https://doi.org/10.1016/j.jmps. 2017.01.010. URL http: / / www. sciencedirect. com/science/article/pii/ s0022509616308997.

[45] Hussein Nassar, Qi-Chang He, and Nicolas Auffray. Willis elastodynamic homogenization theory revisited for periodic media. Journal of the Mechanics and Physics of Solids, 2015.

[46] Sia Nemat-Nasser and Muneo Hori. Micromechanics: overall properties of heterogeneous materials. Elsevier, 2013.

[47] A N Norris, A L Shuvalov, and A A Kutsenko. Analytical formulation of threedimensional dynamic homogenization for periodic elastic systems. Proc. R. Soc. London A Math. Phys. Eng. Sci., 2012. ISSN 1364-5021. doi: 10.1098/rspa.2011.0698. URL http://rspa.royalsocietypublishing.org/content/early/2012/ $02 / 14$ /rspa.2011.0698.

[48] René Pernas-Salomón and Gal Shmuel. Dynamic homogenization of composite and locally resonant flexural systems. J. Mech. Phys. Solids, 119:43-59, 2018. ISSN 0022-5096. doi: https://doi.org/10.1016/j.jmps.2018.06.011. URL http://www. sciencedirect . com/science/article/pii/s0022509618302503.

[49] René Pernas-Salomón and Gal Shmuel. Symmetry breaking creates electro-momentum coupling in piezoelectric metamaterials. Journal of the Mechanics and Physics of Solids, 134:103770, 2020. ISSN 0022-5096. doi: https://doi.org/10.1016/j.jmps.2019. 103770. URL http://www.sciencedirect.com/science/article/pii/ S 0022509619306386.

[50] René Pernas-Salomón and Gal Shmuel. Fundamental Principles for Generalized Willis Metamaterials. Phys. Rev. Applied, 14:064005, Dec 2020. doi: 10.1103/PhysRevApplied.14. 064005. URL https://link.aps.org/doi/10.1103/PhysRevApplied.14. 064005 . 
[51] René Pernas-Salomón, Michael R. Haberman, Andrew N. Norris, and Gal Shmuel. The electromomentum effect in piezoelectric willis scatterers. Wave Motion, page 102797, 2021. doi: https://doi.org/10.1016/j.wavemoti.2021.102797. URL https://www . sciencedirect.com/science/article/pii/s0165212521000950.

[52] Marie-Fraise Ponge, Olivier Poncelet, and Daniel Torrent. Dynamic homogenization theory for nonlocal acoustic metamaterials. Extrem. Mech. Lett., 12:71-76, 2017. ISSN 2352-4316. doi: https://doi.org/10.1016/j.eml.2016.10.006. URL http://www. sciencedirect . com/science/article/pii/s2352431616300979.

[53] Bogdan-Ioan Popa, Yuxin Zhai, and Hyung-Suk Kwon. Broadband sound barriers with bianisotropic metasurfaces. Nature Communications, 9(1):5299, 2018. doi: 10.1038/ s41467-018-07809-3. URL https: / / doi .org/10 .1038/s41467-018-07809-3.

[54] Li Quan, Younes Ra'di, Dimitrios L Sounas, and Andrea Alù. Maximum Willis Coupling in Acoustic Scatterers. Phys. Rev. Lett., 120(25):254301, 2018. doi: 10.1103/PhysRevLett. 120.254301. URL https:// link.aps.org/doi/10.1103/PhysRevLett. 120 . 254301.

[55] G Shmuel. Electrostatically tunable band gaps in finitely extensible dielectric elastomer fiber composites. Int. J. Solids Struct., 50(5):680-686, 2013. ISSN 00207683. doi: 10.1016/j. ijsolstr.2012.10.028.

[56] A. L. Shuvalov, A. A. Kutsenko, A. N. Norris, and O Poncelet. Effective Willis constitutive equations for periodically stratified anisotropic elastic media. Proc. R. Soc. London A Math. Phys. Eng. Sci., 467(2130):1749-1769, 2011. ISSN 1364-5021. doi: 10.1098/ rspa.2010.0389. URL http: / / rspa.royalsocietypublishing . org/content/ $467 / 2130 / 1749$.

[57] Caleb F Sieck, Andrea Alù, and Michael R Haberman. Origins of Willis coupling and acoustic bianisotropy in acoustic metamaterials through source-driven homogenization. Phys. Rev. B, 96(10):104303, 2017. doi: 10.1103/PhysRevB.96.104303. URL https:// I ink . aps . org/doi/10.1103/PhysRevB.96.104303.

[58] M M Sigalas and E N Economou. Attenuation of multiple-scattered sound. Europhys. Lett., 36(4):241-246, 1996. doi: 10.1209/epl/i1996-00216-4.

[59] C. R. Simovski. Material parameters of metamaterials (a review). Optics and Spectroscopy, 107(5):726, 2009. doi: 10.1134/S0030400X09110101. URL https : / / doi . org/10 . $1134 / \mathrm{S} 0030400 \times 09110101$. 
[60] C R Simovski. On electromagnetic characterization and homogenization of nanostructured metamaterials. Journal of Optics, 13(1):013001, nov 2010. doi: 10.1088/2040-8978/13/1/ 013001. URL https://doi.org/10.1088/2040-8978/13/1/013001.

[61] David R. Smith and John B. Pendry. Homogenization of metamaterials by field averaging (invited paper). J. Opt. Soc. Am. B, 23(3):391-403, Mar 2006. doi: 10.1364/JOSAB.23.000391. URL http: / / josab.osa.org/abstract. cfm?URI=josab-23-3-391.

[62] A Sridhar, V G Kouznetsova, and M G D Geers. A general multiscale framework for the emergent effective elastodynamics of metamaterials. J. Mech. Phys. Solids, 111:414-433, 2018. ISSN 0022-5096. doi: https://doi.org/10.1016/j.jmps.2017.11.017. URL http:// www.sciencedirect.com/science/article/pii/s0022509617306245.

[63] A Srivastava and S Nemat-Nasser. On the limit and applicability of dynamic homogenization. Wave Motion, 51(7):1045-1054, 2014. ISSN 0165-2125. doi: https://doi.org/10. 1016/j.wavemoti.2014.04.003. URL http://www.sciencedirect.com/science/ article/pii/s0165212514000614.

[64] Ankit Srivastava. Causality and passivity in elastodynamics. Proceedings of the Royal Society of London A: Mathematical, Physical and Engineering Sciences, 471 (2180), 2015. ISSN 1364-5021. doi: 10.1098/rspa.2015.0256. URL http://rspa. royalsocietypublishing.org/content/471/2180/20150256.

[65] Ankit Srivastava. Elastic metamaterials and dynamic homogenization: a review. International Journal of Smart and Nano Materials, 6(1):41-60, 2015.

[66] Ankit Srivastava. Causality and passivity: From electromagnetism and network theory to metamaterials. Mechanics of Materials, 154:103710, 2021. ISSN 0167-6636. doi: https:// doi.org/10.1016/j.mechmat.2020.103710. URL https: //www. sciencedirect.com/ science/article/pii/s0167663620307377.

[67] Luc Tartar. The general theory of homogenization: a personalized introduction, volume 7. Springer Science \& Business Media, 2009.

[68] Daniel Torrent and José Sánchez-Dehesa. Multiple scattering formulation of two-dimensional acoustic and electromagnetic metamaterials. New Journal of Physics, 13(9):093018, sep 2011. doi: 10.1088/1367-2630/13/9/093018. URL https://doi.org/10.1088\% $2 \mathrm{~F} 1367-2630 \div 2 \mathrm{~F} 13 \div 2 \mathrm{~F} 9 \% 2 \mathrm{~F} 093018$. 
[69] Daniel Torrent, Yan Pennec, and Bahram Djafari-Rouhani. Resonant and nonlocal properties of phononic metasolids. Phys. Rev. B, 92(17):174110, 2015. doi: 10.1103/PhysRevB.92. 174110. URL https://1ink.aps.org/doi/10.1103/PhysRevB.92.174110.

[70] Daniel Torrent, Olivier Poncelet, and Jean-Chirstophe Batsale. Nonreciprocal thermal material by spatiotemporal modulation. Phys. Rev. Lett., 120:125501, Mar 2018. doi: 10.1103/PhysRevLett.120.125501. URL https://1ink.aps.org/doi/10.1103/ PhysRevLett.120.125501.

[71] Martin Wegener. Metamaterials beyond optics. Science, 342(6161):939-940, 2013. ISSN 0036-8075. doi: 10.1126/science.1246545. URL https://science.sciencemag • org/content/342/6161/939.

[72] J R Willis. Variational principles for dynamic problems for inhomogeneous elastic media. Wave Motion, 3(1):1-11, 1981. ISSN 0165-2125. doi: https://doi.org/10. 1016/0165-2125(81)90008-1. URL http://www.sciencedirect.com/science/ article/pii/0165212581900081.

[73] J R Willis. The nonlocal influence of density variations in a composite. Int. J. Solids Struct., 21(7):805-817, 1985. ISSN 0020-7683. doi: https://doi.org/10.1016/0020-7683(85) 90084-8. URL http://wWw.sciencedirect.com/science/article/pii/ 0020768385900848 .

[74] J R Willis. Continuum Micromechanics. chapter Dynamics o, pages 265-290. SpringerVerlag New York, Inc., New York, NY, USA, 1997. ISBN 3-211-82902-4. URL http: //dl.acm.org/citation.cfm?id=265753.265760.

[75] J R Willis. Effective constitutive relations for waves in composites and metamaterials. Proc. R. Soc. London A Math. Phys. Eng. Sci., 467(2131):1865-1879, 2011. ISSN 1364-5021. doi: 10.1098/rspa.2010.0620. URL http://rspa.royalsocietypublishing . org/content/467/2131/1865.

[76] J R Willis. Some thoughts on dynamic effective properties-a working document. 2013. URL https://arxiv.org/abs/1311.3875.

[77] J. R. Willis. From statics of composites to acoustic metamaterials. Philosophical Transactions of the Royal Society A: Mathematical, Physical and Engineering Sciences, 377(2156), 2019.

[78] John R Willis. Variational and related methods for the overall properties of composites. Advances in applied mechanics, 21:1-78, 1981. 
[79] John R. Willis. The construction of effective relations for waves in a composite. Comptes Rendus Mécanique, 340(4):181 - 192, 2012. ISSN 1631-0721. doi: https://doi.org/10.1016/j. crme.2012.02.001. URL http://www.sciencedirect.com/science/article/ pii/S1631072112000381. Recent Advances in Micromechanics of Materials.

[80] J.R. Willis. A comparison of two formulations for effective relations for waves in a composite. Mechanics of Materials, 47:51 - 60, 2012. ISSN 0167-6636. doi: https://doi.org/10. 1016/j.mechmat.2011.12.008. URL http://www.sciencedirect.com/science/ article/pii/s0167663612000105.

[81] Yuxin Zhai, Hyung-Suk Kwon, and Bogdan-Ioan Popa. Active willis metamaterials for ultracompact nonreciprocal linear acoustic devices. Phys. Rev. B, 99:220301, Jun 2019. doi: 10.1103/PhysRevB.99.220301. URL https://link.aps.org/doi/10.1103/ PhysRevB. 99.220301. 